\title{
Curcumin Protects Against Cognitive Impairments in a Rat Model of Chronic Cerebral Hypoperfusion Combined with Diabetes Mellitus by Suppressing Neuroinflammation, Apoptosis, and Pyroptosis
}

\author{
Yaling Zheng \\ Shanghai Jiao Tong University Affiliated Sixth People's Hospital \\ Jiawei Zhang \\ Shanghai Jiao Tong University Affiliated Sixth People's Hospital \\ Yao Zhao \\ Shanghai Jiao Tong University Affiliated Sixth People's Hospital \\ Yaxuan Zhang \\ Shanghai Jiao Tong University Affiliated Sixth People's Hospital \\ Xiaojie Zhang \\ Shanghai Jiao Tong University Affiliated Sixth People's Hospital \\ Jian Guan \\ Shanghai Jiao Tong University Affiliated Sixth People's Hospital \\ Yu Liu \\ Shanghai Eighth People's Hospital \\ Jianliang Fu ( $\sim$ fujianliang@163.com ) \\ Shanghai Jiao Tong University Affiliated Sixth People's Hospital
}

\section{Research}

Keywords: Curcumin, Chronic cerebral hypoperfusion, Diabetes, Neuroinflammation, Apoptosis, Pyroptosis

Posted Date: November 20th, 2020

DOI: https://doi.org/10.21203/rs.3.rs-111414/v1

License: (c) (1) This work is licensed under a Creative Commons Attribution 4.0 International License. Read Full License 


\section{Abstract}

\section{Background}

Chronic cerebral hypoperfusion ( $\mathrm{CCH}$ ) is regarded as a high-risk factor for cognitive decline in vascular dementia (VaD). We have previously shown that diabetes mellitus (DM) synergistically promotes $\mathrm{CCH}$ induced cognitive dysfunction via exacerbating neuroinflammation. Furthermore, curcumin has been shown to exhibit anti-inflammatory and neuroprotective activities. However, the effects of curcumin on $\mathrm{CCH}$-induced cognitive impairments in DM have remained unknown.

\section{Methods}

Rats were fed with a high-fat diet (HFD) and injected with low-dose streptozotocin (STZ), followed by bilateral common carotid artery occlusion (BCCAO), to model DM and $\mathrm{CCH}$ in vivo. After BCCAO, curcumin $(50 \mathrm{mg} / \mathrm{kg}$ ) was administered intraperitoneally every two days for eight weeks to evaluate its therapeutic effects. Additionally, mouse BV2 microglial cells were exposed to hypoxia and high glucose to model $\mathrm{CCH}$ and DM pathologies in vitro.

\section{Results}

Curcumin treatment significantly improved $\mathrm{DM} / \mathrm{CCH}$-induced cognitive deficits and attenuated neuronal cell death. Molecular analysis revealed that curcumin exerted protective effects via suppressing neuroinflammation induced by microglial activation, regulating the triggering receptor expressed on myeloid cells 2 (TREM2)/toll-like receptor 4 (TLR4)/nuclear factor-KB (NF-KB) pathway, alleviating apoptosis, and reducing nod-like receptor protein 3 (NLRP3)-dependent pyroptosis.

\section{Conclusions}

Taken together, our findings suggest that curcumin represents a promising therapy for $\mathrm{DM} / \mathrm{CCH}$-induced cognitive impairments.

\section{Introduction}

As the second-leading cause of dementia following Alzheimer's disease (AD), vascular dementia (VaD) is characterized by memory decline and cognitive dysfunction ${ }^{[1]}$. Chronic cerebral hypoperfusion $(\mathrm{CCH})$, a chronic state of reduced cerebral blood flow, has been associated with the pathological processes of $\mathrm{VaD}$ resulting from disorders affecting the cerebral vascular system, including hypertension, diabetes, generalized atherosclerosis, and smoking ${ }^{[2-5]}$. As a chronic metabolic disease, the prevalence of diabetes mellitus (DM) has increased worldwide ${ }^{[6]}$. Notably, epidemiological studies have established an increased risk of dementia among individuals with $\mathrm{DM}^{[7,8]}$. Accumulating evidence has also revealed that $\mathrm{DM}$ interacts with $\mathrm{CCH}$ to promote the pathophysiological progression of neurodegenerative disorders $[9,10]$. 
Furthermore, our previous studies have demonstrated that DM synergistically promotes $\mathrm{CCH}$-induced cognitive dysfunction ${ }^{[11]}$.

At present, the potential mechanisms of $\mathrm{CCH}$-induced $\mathrm{VaD}$ are related to hippocampal neuronal injury, white matter damage, neuroinflammation, and oxidative stress. Among these factors, neuroinflammation has recently received increased attention ${ }^{[12-15]}$. Neuroinflammation mainly occurs through the activation of microglia and subsequent production of neurotoxic pro-inflammatory cytokines, which further induce neuronal damage and even neuronal death, resulting in brain damage ${ }^{[16,17]}$. A recent study found that in a rat model of $\mathrm{CCH}$, synaptic plasticity was significantly impaired along with an increase in microglial activation, thus contributing to cognitive deficits ${ }^{[18]}$. A number of signaling molecules, including toll-like receptor 4 (TLR4)/nuclear factor-KB (NF-KB) and triggering receptor expressed on myeloid cells 2 (TREM2), are essential for modulation of microglial activation and neuroinflammation. TREM2 is an important innate immune receptor that is expressed uniquely on microglia in the central nervous system and participates in down-regulating neuroinflammation ${ }^{[19]}$. Consistently, our previous study also demonstrated that there was an imbalance between microglial TLR4- and TREM2-mediated microglial polarization and neuroinflammation in lipopolysaccharide (LPS)-treated BV2 cells ${ }^{[20]}$. However, the role of the TREM2/TLR4 pathway in DM/CCH-induced pathologies has remained unclear.

As previously reported, apoptosis is thought to be involved in the occurrence and development of $\mathrm{VaD}^{[21-}$ 23]. Some studies have shown that increased activation of mitochondrial apoptotic markers (e.g., caspase 3) leads to destruction of the blood-brain barrier, inflammation, and oxidative stress, and eventually results in brain damage ${ }^{[22,24,25]}$. Moreover, pyroptosis, a pro-inflammatory form of programmed cell death, also contributes to the pathogeneses of neurodegenerative diseases such as AD and Parkinson's disease (PD) ${ }^{[26,27]}$. Nod-like receptor protein 3 (NLRP3) inflammasome is known to contribute to upstream signaling of pyroptosis ${ }^{[28-30]}$. After activation, NLRP3 inflammasome triggers cleavage and activation of caspase 1 , which mediates pyroptosis ${ }^{[28]}$. However, whether apoptosis and pyroptosis promote the progression of $\mathrm{VaD}$ in $\mathrm{DM}$ patients remains unclear.

Curcumin, derived from the rhizome of Curcuma longa Linn, is a bright yellow spice that shows strong anti-inflammatory, anti-oxidant, and anti-tumor activities ${ }^{[31,32]}$. Many studies have reported neuroprotective effects of curcumin in neurodegenerative diseases, including AD, PD, and multiple sclerosis $^{[33-36]}$. Our previous study has also demonstrated a protective effect of curcumin on LPSinduced neuroinflammation in vitro ${ }^{[20]}$. However, whether curcumin exerts neuroprotective effects on $\mathrm{DM} / \mathrm{CCH}$-induced pathologies has remained unknown.

In the present study, to investigate the protective effects and mechanisms of curcumin, we established an in-vivo rat model of DM/CCH induced by a high-fat diet (HFD) and streptozotocin (STZ) treatment, followed by bilateral common carotid artery occlusion (BCCAO). Additionally, we established an in-vitro model of $\mathrm{DM} / \mathrm{CCH}$-induced pathologies in mouse BV2 microglia exposed to hypoxia and high glucose . 


\section{Methods}

\subsection{Animals}

Healthy adult male Sprague-Dawley (SD) rats (six weeks old, 160-180 g) were used in the present study. Rats were housed under a controlled temperature $\left(23 \pm 1^{\circ} \mathrm{C}\right)$ and a 12 -h light/dark cycle, and were provided food and water ad libitum. All experiments were approved by the Institutional Animal Care and Use Committee of Shanghai Jiao Tong University.

\subsection{Rat model of DM and $\mathrm{CCH}$}

After one week of acclimatization, the rats were randomly divided into four groups: sham surgery (Sham group), sham + curcumin (Cur group), DM + two-step BCCAO (DM/CCH group), and DM + BCCAO + curcumin ( $\mathrm{DM} / \mathrm{CCH}+$ Cur group) ( $\mathrm{n}=9$ per group). Our $\mathrm{DM}$ rat model was established according to the methods reported in our previous studies ${ }^{[11,37]}$. Briefly, the diabetic rats were fed with a HFD $(60 \%$ fat, $20 \%$ carbohydrate, and $20 \%$ protein) while non-diabetic rats were fed with a low-fat diet (LFD) ( $10 \%$ fat, $70 \%$ carbohydrate and $20 \%$ protein). After six weeks on these feeding regimens, rats fed with a HFD were intraperitoneally administered with a single dose of STZ $(30 \mathrm{mg} / \mathrm{kg})$. One week after the STZ injection, $1.0 \mathrm{~mL}$ of blood was collected from the tail vein of each injected rat. Rats were considered diabetic when their blood glucose reached $16.7 \mathrm{mM}$ one week after injection of STZ. Two weeks after injection of STZ, rats received sham surgery or two-step BCCAO according to a previously described procedure ${ }^{[38]}$. Briefly, rats were anesthetized by an intraperitoneal injection of chloral hydrate $(350 \mathrm{mg} / \mathrm{kg})$ and were then fixed in a supine position. A midline cervical incision was made, and both the left and the right common carotid arteries were carefully isolated from the vagus nerve. In the $\mathrm{DM} / \mathrm{CCH}$ and $\mathrm{DM} / \mathrm{CCH}+\mathrm{Cur}$ groups, the bilateral common carotid arteries were permanently ligated with \#4 surgical thread, and the left common carotid artery was occluded first; then, one week later, the right common carotid artery was occluded. The Sham and the Cur groups underwent the same surgical procedure without BCCAO. During the surgery, body temperature was maintained at approximately $37^{\circ} \mathrm{C}$ by a heating pad. Of the 18 rats in the BCCAO groups, two rats $(11 \%)$ died on the first day after operation, which may have been due to excessive anesthesia and/or surgical injury.

\subsection{Curcumin preparation and treatment}

Curcumin $(0.1 \mathrm{~g})$ was dissolved in $1 \mathrm{~mL}$ of dimethylsulfoxide (DMSO) and diluted with $9 \mathrm{~mL}$ of oil. In the Cur and $\mathrm{DM} / \mathrm{CCH}+$ Cur groups, each rat was intraperitoneally injected with $1.5 \mathrm{~mL}$ of curcumin (50 $\mathrm{mg} / \mathrm{kg}$ ) every two days for eight weeks after BCCAO.

\subsection{Morris water maze (MWM)}

Spatial learning and memory abilities of all rats were evaluated via the Morris water maze (MWM) at eight weeks after BCCAO was performed. The MWM was carried out in a large circular black pool with a diameter of $180 \mathrm{~cm}$ and a height of $50 \mathrm{~cm}$, which was filled with water $\left(24 \pm 1^{\circ} \mathrm{C}\right)$. Charcoal-black ink was added to make the water opaque. The pool was divided into four equal quadrants, and a movable hidden 
platform ( $10 \mathrm{~cm}$ in diameter) was placed $2 \mathrm{~cm}$ below the surface in the second quadrant of the MWM. The MWM included two steps. The first step was the place navigation test, which was conducted four times a day for five consecutive days. Each rat was gently placed in the water from a different quadrant facing the inner wall of the pool. Once within the MWM, rats were allowed to swim for up to $60 \mathrm{~s}$. Once each rat reached the platform, it was allowed to remain on the platform for $20 \mathrm{~s}$. If the rat failed to find the hidden platform within $60 \mathrm{~s}$, it was gently placed on the platform for $20 \mathrm{~s}$. In each test, the time that the rat took to reach the hidden platform was recorded as the escape latency. The second step of the MWM was the spatial probe test, which was performed on the sixth day and included removal of the hidden platform. During the spatial probe test, each rat was placed in the opposite quadrant to that of the target quadrant and allowed to swim freely for $60 \mathrm{~s}$. The time spent in the target quadrant, the number of times crossing the area in which the platform was located previously, and the average swimming speed were measured.

\subsection{Tissue preparation}

All rats were sacrificed after completing the MWM. Brains were perfused with $0.9 \%$ cold saline and were immediately separated into two hemispheres. One of the hemispheres was rapidly frozen in liquid nitrogen for tissue biochemical analysis, while the other hemisphere was fixed in paraformaldehyde (4\%) at $4{ }^{\circ} \mathrm{C}$ for $24 \mathrm{~h}$ for histological or immunohistochemical detection.

\subsection{Immunostaining}

In order to carry out histological analysis, brain tissue sections were dewaxed, rehydrated, and stained with hematoxylin and eosin (HE, purchased from Servicebio) according to the manufacturer's instructions. Light microscopy was used to observe the histomorphology of neurons. For immunofluorescent staining, brain slices were incubated with blocking buffer ( $3 \%$ donkey serum, 1\% BSA, and $0.3 \%$ Triton $\mathrm{X}-100$ in phosphate-buffered saline [PBS]) at room temperature for $1 \mathrm{~h}$ after returning to room temperature. Then, the sections were incubated with the following primary antibodies overnight at

$4{ }^{\circ} \mathrm{C}$ : goat anti-Iba1 (1:500, Abcam) and rabbit anti-NeuN (1:500, Abcam). After being washed three times in PBS, the sections were incubated with secondary antibodies at room temperature for $1 \mathrm{~h}$ in the dark. For labeling cell nuclei, sections were incubated in DAPI and an anti-fluorescent quenching agent after being washed three times in PBS. The sections were then visualized under an Olympus microscope (IX53).

\subsection{Detection of apoptosis}

To examine neuronal apoptosis in the hippocampus, terminal deoxynucleotidyl transferase dUTP nickend labeling (TUNEL) was performed. The TUNEL staining kit was purchased from Beyotime (Shanghai, China). The sections were fixed in paraformaldehyde and were then subjected to various reagents of the TUNEL staining kit, according to the manufacturer's instructions. Images were visualized under an Olympus microscope (IX53).

\subsection{Cell culture and treatments}


Mouse BV2 microglial cells (purchased from Shanghai Zhong Qiao Xin Zhou Biotechnology Co. Ltd) were cultured in eagle's minimum essential medium (EMEM) containing $10 \%$ fetal bovine serum and $1 \%$ penicillin/streptomycin at $37^{\circ} \mathrm{C}$ with $5 \% \mathrm{CO}$. When treated with high glucose, D-glucose was added to the conventional medium to yield a final concentration of $30 \mathrm{mM}$ in vitro. During hypoxia treatment, the cells were placed in an 02/N2/CO2 incubator and exposed to $3 \% 02$ for $24 \mathrm{~h}$. The experiments in vitro consisted of the following four groups: (1) cells that were cultured without any treatment (control group); (2) cells that were pretreated with curcumin $(10 \mu \mathrm{M})$ for $2 \mathrm{~h}$ under normal culture conditions (Cur group); (3) cells that were exposed to high glucose $(30 \mathrm{mM})$ for $24 \mathrm{~h}$ followed by hypoxia ( $3 \% 02)$ for $24 \mathrm{~h}$ (HG/HP group); and (4) cells that were pretreated with curcumin $(10 \mu \mathrm{M})$ for $2 \mathrm{~h}$ under high glucose combined with hypoxic conditions as described above (HG/HP + Cur group).

\subsection{Cell viability assays}

Cell viabilities were determined by cell counting kit 8 (CCK8) assays. Briefly, BV2 cells were seeded in 96well plates at a density of 4,000 cells per well and were exposed to different conditions overnight according to their designated groups. After incubation with CCK8 reagent $(10 \mu \mathrm{L})$ for $2 \mathrm{~h}$, the optical density (OD) within each well was read on a microplate reader at a wavelength of $450 \mathrm{~nm}$.

\subsection{Flow cytometry}

Cells were cultured in six-well plates and exposed to different conditions according to their designated groups. The cells were digested with trypsin and were then centrifuged. After being washed three times with cold PBS, the cells were resuspended with $100 \mu \mathrm{L}$ of binding buffer. Then, $5 \mu \mathrm{L}$ of annexin V-FITC and $5 \mu \mathrm{L}$ of PI were added to react for $15 \mathrm{~min}$ at room temperature in the dark. Then, $400 \mu \mathrm{L}$ of binding buffer was added to the cells and mixed softly, and then the cells were subjected to flow cytometry (CytoFLEX LX, USA).

\subsection{Quantitative real-time PCR (qRT-PCR)}

Total RNA was isolated from the brain tissues and cells using an RNAeasy animal RNA isolation kit (Beyotime, Shanghai, China) according to the manufacturer's instructions. Isolated RNA was reversetranscribed to synthesize cDNA using a cDNA Synthesis kit (Takara, Japan). Then, quantitative real-time PCR (qRT-PCR) was performed using TB Green Premix Ex Taq II (Tli RNaseH Plus) with an Applied Biosystems QuantStudio 7 Real-Time PCR System. All primer sequences that we used are listed in Table 1.

\subsection{Western blot analysis}

Total proteins were extracted from brain tissues or cells using lysates containing RIPA buffer supplement with protease and phosphatase inhibitors. The protein concentration of each sample was determined using bicinchoninic acid (BCA) assays. Then, the proteins were separated by sodium-dodecyl-sulfate 
polyacrylamide gel electrophoresis (SDS-PAGE) and were then electro-transferred onto a polyvinylidene fluoride (PVDF) membrane. After blocking with $5 \%$ milk or BSA, the membrane was incubated at $4{ }^{\circ} \mathrm{C}$ overnight with the following primary antibodies: TREM2, 1:500; TLR4, 1:200; NF-KB, 1:1000; p-NF- KB, 1:1000; Bax, 1:1000; Bcl2, 1:1000; cleaved-caspse3, 1:1000; NLRP3, 1:1000; ASC, 1:1000; caspase1, 1:1000; GSDMD-N, 1:1000; IL-18, 1:1000; and actin, 1:1000. After three washes (for 10 min each) with TBST buffer, the membrane was incubated with secondary anti-rabbit or anti-mouse antibodies for $1 \mathrm{~h}$ at room temperature. The protein bands were visualized using enhanced chemiluminescence.

\subsection{Statistical analysis}

Data are expressed as the mean \pm standard error of the mean (SEM). Comparisons among different groups were performed using one-way analysis of variance (ANOVA) with SPSS 22.0. Repeated-measures multivariate analysis of variance was used to evaluate the results of the MWM. A P $<0.05$ was considered statistically significant.

\section{Results}

\subsection{Curcumin alleviates DM/CCH-induced impairments in spatial learning and memory in rats}

Eight weeks after BCCAO, the MWM was used to investigate whether curcumin could alleviate DM/CCHinduced impairments in spatial learning and memory. In the hidden platform test, the DM/CCH group exhibited a significantly-prolonged escape latency compared to that of the Sham group since the second day on the MWM, especially on days 4-5 (Fig. 1B). However, curcumin treatment significantly mitigated the poor spatial learning performance resulting from $\mathrm{DM} / \mathrm{CCH}$, whereas curcumin did not induce any changes in the learning/memory performances of the Sham and Cur groups. In the probe test, the rats in the $\mathrm{DM} / \mathrm{CCH}$ group exhibited fewer crossings over the location of the original platform, less time spent in the target quadrant, and more chaotic trajectories than those of the Sham and Cur groups. However, curcumin treatment dramatically mitigated these spatial memory impairments caused by $\mathrm{DM} / \mathrm{CCH}$

(Fig. 1C, D, and F). There were no differences in the swimming speeds among any of the groups (Fig. 1E). Taken together, these results indicate that curcumin significantly alleviated DM/CCH-induced impairments in spatial learning and memory.

\subsection{Curcumin attenuates neuronal death in $\mathrm{DM} / \mathrm{CCH}$ rats}

$\mathrm{DM} / \mathrm{CCH}$-induced neuronal death in the hippocampus was examined by $\mathrm{HE}$ and NeuN staining. $\mathrm{HE}$ staining revealed normal morphologies and clear boundaries of hippocampal neurons in the Sham and the Cur groups, while the DM/CCH group exhibited abnormal morphologies of shrunken neurons with dark-stained nuclei, and even missing/lost neurons. Surprisingly, curcumin treatment partially attenuated such neuronal damage (Fig. 2A). Moreover, curcumin treatment effectively restored the DM/CCH-induced decreased number of neurons in the $\mathrm{CA} 1, \mathrm{CA} 3$, and dentate gyrus $(\mathrm{DG})$ regions of the hippocampus 
(Fig. 2B). These findings suggest that curcumin attenuated DM/CCH-induced neuronal death in the CA1, CA3, and DG regions of the hippocampus.

\subsection{Curcumin inhibits $\mathrm{DM} / \mathrm{CCH}$-induced neuroinflammation in the rat hippocampus}

Previous studies have reported that neuroinflammation plays a vital role in the pathology of $\mathrm{VaD}^{[14,39-41]}$. Microglia are resident immune cells within the central nervous system and play an important role in the initiation and transmission of inflammatory responses. Thus, we investigated whether curcumin could suppress DM/CCH-induced microglial activation and production of pro-inflammatory cytokines. Iba1 was used to label microglia. As shown in Fig. 3A, activated microglia were rarely detected in the CA1 region of the hippocampus in the Sham and Cur groups, while the numbers of Iba1-positive cells were increased dramatically following DM/CCH in the hippocampus. However, curcumin inhibited this DM/CCH-induced overactivation of microglia, as microglial density was reduced compared to that in the DM/CCH group. We further detected the expression of pro-inflammatory (IL-1 $\beta, I L-6$, TNF-a, iNOS, COX2) and antiinflammatory (Arg-1, IL-4, IL-10) cytokines in each group via qRT-PCR. As shown in Fig. 3B, IL-1 $\beta$, IL-6, and TNF-a levels were increased whereas Arg-1, IL-4, and IL-10 levels were decreased in the DM/CCH group compared with those in the Sham group. However, curcumin treatment significantly reduced this $\mathrm{DM} / \mathrm{CCH}$-induced inflammatory response, as the levels of pro-inflammatory cytokines were decreased while those of anti-inflammatory cytokines were increased compared to those in the DM/CCH group. Similarly, our in-vitro model confirmed that compared with those in the Control group, hypoxia combined with high glucose markedly increased IL-1 $\beta$, IL-6, TNF-a, iNOS, and COX2 levels while reducing Arg-1 levels. However, curcumin effectively reversed high-glucose/hypoxia-induced changes in expression levels of pro-inflammatory and anti-inflammatory cytokines (Fig. 3C). Meanwhile, there were no differences in levels of inflammatory cytokines between the Sham and Cur groups, either in vivo or in vitro. These results demonstrate that curcumin inhibited DM/CCH-induced and high-glucose/hypoxiainduced microglial activation and pro-inflammatory cytokine production.

\subsection{Curcumin regulates TREM2/TLR4/NF-KB signaling in $\mathrm{DM} / \mathrm{CCH}$ rats and high-glucose/hypoxia-treated microglia}

TLR4 and TREM2 are microglial membrane-bound receptors that play important roles in inflammatory signaling pathways ${ }^{[42,43]}$. Additionally, NF-KB activation induces the release of pro-inflammatory cytokines $^{[44]}$. Therefore, in our present study, we first measured protein levels of TREM2, TLR4, and NF-KB via Western blotting in vivo. As shown in Fig. 4A and B, the protein levels of TREM2, TLR4, and phosphorylated NF-KB p65 (p-NF-KB p65) were increased in the DM/CCH group compared with those in the Sham group. Interestingly, curcumin treatment effectively inhibited TLR4 and p-NF-kB p65 levels, whereas it further increased TREM2 levels. No significant differences were found in terms of NF-KB p65 levels. Furthermore, our results in vitro were similar to those in vivo. As shown in Fig. $4 C$ and D, the expression levels of TREM2, TLR4, and p-NF-KB p65 were significantly increased in the highglucose/hypoxia-treated group compared with those in the Control group. After curcumin treatment, TLR4 
and p-NF-KB p65 levels were dramatically decreased while TREM2 levels were further increased. Meanwhile, we also measured mRNA levels of TREM2 and TLR4 both in vivo and in vitro. As shown in Fig. 4E and F, our results of mRNA levels were consistent with those of protein levels. There were no differences in the measured mRNA or protein levels between the Sham (Control) and Cur groups. Therefore, these results suggest that curcumin inhibited neuroinflammation by regulating the TREM2/TLR4/NF-KB pathway in both DM/CCH rats in vivo and high-glucose/hypoxia-treated microglia in vitro.

\subsection{Curcumin suppresses apoptosis in $\mathrm{DM} / \mathrm{CCH}$ rats and high-glucose/hypoxia-treated microglia}

As previously reported, apoptosis is involved in the development of $\mathrm{VaD}^{[45,46]}$. Hence, we first investigated the levels of apoptosis-related proteins (Bax, Bcl2, and cleaved-caspase3) via Western blotting in vivo. As shown in Fig. $5 \mathrm{~A}$ and $\mathrm{B}, \mathrm{DM} / \mathrm{CCH}$ rats exhibited higher levels of Bax and cleaved-caspase 3 and lower levels of $\mathrm{Bcl} 2$ compared to those in the Sham group, whereas these changes in protein levels were reversed by curcumin. Furthermore, our results in vitro were consistent with those in vivo. As shown in Fig. 5C and D, curcumin effectively reversed high-glucose/hypoxia-induced overexpression of Bax and cleaved-caspase3 and restored Bcl2 levels in microglia in vitro. Furthermore, qRT-PCR confirmed that curcumin reduced the mRNA levels of Bax and cleaved-caspase 3 while increasing Bcl2 mRNA levels both in vivo and in vitro (Fig. 5E and F). In contrast, there were no significant differences in any measured mRNA or protein levels between the Sham (Control) and Cur groups.

Next, we used TUNEL staining to examine neuronal apoptosis in vivo, and Annexin-FITC/PI double staining as well as CCK8 assays to examine microglial death in vitro. As shown in Fig. 6A, DM/CCH rats exhibited a higher number of TUNEL-positive cells in the CA1 region of the hippocampus compared with that in the Sham group. Strikingly, curcumin treatment significantly reduced the number of TUNELpositive cells. Annexing-FITC/PI double staining revealed that curcumin effectively suppressed highglucose/hypoxia-induced microglial apoptosis (Fig. 6B and C). Our results also demonstrated that cellular survival rates were decreased dramatically in response to hypoxia and high glucose, while curcumin treatment effectively restored cellular viability (Fig. 6D). There were no significant differences in terms of apoptosis between the Sham (Control) and Cur groups. Taken together, these findings suggest that curcumin suppressed apoptosis following $\mathrm{DM} / \mathrm{CCH}$ in vivo or high-glucose/hypoxia in vitro.

\subsection{Curcumin mitigates NLRP3-dependent pyroptosis in $\mathrm{DM} / \mathrm{CCH}$ rats and high-glucose/hypoxia-treated microglia}

Accumulating evidence has demonstrated that NLRP3-dependent pyroptosis is involved in the progression of neurodegenerative diseases ${ }^{[47,48]}$. Therefore, Western blotting and qRT-PCR were used to detect protein and mRNA levels, respectively, of NLRP3, apoptosis-associated speck-like protein containing a CARD (ASC, which is an adaptor protein connecting pattern-recognition receptors and procaspase1), cleaved-caspase1, cleavage of gasdermin D (GSDMD-N, a protein substrate of caspase1 that has been identified as an executive molecule in pyroptosis), and IL-18 in vivo and in vitro. As shown in 
Fig. 7A and $B$, curcumin treatment significantly reduced DM/CCH-induced overexpression of NLRP3, ASC, cleaved-caspase1, GSDMD-N, and IL-18 proteins. Our results in vitro were similar to those in vivo, as shown in Fig. 7C and D. The protein levels of microglial NLRP3, ASC, cleaved-caspase1, GSDMD-N, and IL-18 were increased after exposure to high glucose and hypoxia, whereas these changes in protein levels reversed by curcumin treatment. Similarly, curcumin effectively attenuated $\mathrm{DM} / \mathrm{CCH}$-induced or highglucose/hypoxia-induced increases in mRNA levels of NLRP3 and IL-18. These findings indicate that curcumin mitigated NLRP3-dependent pyroptosis in DM/CCH rats and high-glucose/hypoxia-treated microglia.

\section{Discussion}

To investigate the protective effects of curcumin on DM/CCH-induced pathologies, our previous studies have successfully established stable models in vivo and in vitro ${ }^{[11,38]}$. Specifically, we subjected rats to a HFD and low-dose STZ combined with BCCAO to model DM/CCH in vivo, whereas we established a corresponding model in vitro by subjecting BV2 microglia to hypoxia and a high-glucose environment. The present study revealed that curcumin treatment effectively protected against $\mathrm{DM} / \mathrm{CCH}$-induced cognitive dysfunction, as well as attenuated neuronal injury and death in the CA1, CA3, and DG regions of the hippocampus. Molecular-biology analysis revealed that the underlying mechanisms of curcumin's protective effects were associated with inhibiting neuroinflammation, regulating the TREM2/TLR4/NF-KB pathway, suppressing neuronal apoptosis, and mitigating NLRP3-dependent pyroptosis.

$\mathrm{CCH}$ plays a pivotal role in the progression of cognitive impairments in $\mathrm{VaD}$, as it can induce neuroinflammation, decrease energy supplementation, contribute to neuronal injury, and impair memory ${ }^{[18,49,50]}$. Importantly, a previous study has demonstrated that diabetes aggravates neuronal apoptosis and accelerates cognitive dysfunction ${ }^{[51]}$. In our present study, in the MWM, the DM/CCH group exhibited impairments in spatial learning and memory, as revealed by a prolonged escape latency, decreased number of crossings over the original platform location, and less time spent in the target quadrant; these findings are consistent with those of previous reports ${ }^{[11,52,53]}$. However, long-term curcumin treatment significantly ameliorated cognitive dysfunction. Moreover, we found that curcumin attenuated DM/CCH-induced neuronal damage, as there was an increase in NeuN-positive cells and HE staining showed a decrease in atrophied neurons and dark-stained nuclei; these results were consistent with our behavioral results in the MWM. Collectively, these findings indicate that curcumin protected against cognitive impairments and attenuated neuronal damage in $\mathrm{DM} / \mathrm{CCH}$ rats.

Accumulating evidence has demonstrated that $\mathrm{CCH}$ induces significant inflammatory responses, including glial activation and excessive production of pro-inflammatory cytokines (e.g., IL-1 $\beta$, IL-6, TNF- $a$ ), which are pathological signatures that are closely related to neuronal damage ${ }^{[54]}$. Moreover, previous studies have also found that DM increases microglial activation and the release of pro-inflammatory cytokines ${ }^{[55,56]}$. Taken together, these findings suggest that neuroinflammation might play a vital role in the occurrence and development of DM combined with $\mathrm{CCH}$. Consistent with these previous results, in our 
present study, we found overactivation of microglia and excessive production of pro-inflammatory cytokines in $\mathrm{DM} / \mathrm{CCH}$ rats. However, curcumin treatment dramatically inhibited neuroinflammation, as indicated by decreased Iba1-positive cells and decreased production of pro-inflammatory cytokines compared to those in $\mathrm{DM} / \mathrm{CCH}$ rats. Our results in vitro also indicated that curcumin reversed highglucose/hypoxia-induced increases in pro-inflammatory cytokines. $\mathrm{CCH}$ causes a cascade of pathological processes in which many different signaling pathways are activated, including the TREM2/TLR4/NF-KB pathway. TLR4, a classic pattern-recognition receptor, is mainly expressed in microglia and is stimulated by its cognate ligands. Following stimulation, TLR4 further activates the NF$\mathrm{KB}$ pathway, which plays an important role in the transcription of pro-inflammatory cytokines, including IL-1 $\beta$, IL-6, and TNF-a ${ }^{[57]}$. Recently, several studies have demonstrated that TREM2 downregulates TLR4 signaling ${ }^{[19,58,59]}$. As a membrane-bound receptor mainly expressed in microglia of the central nervous system, TREM2 has been confirmed to regulate the primary functions of microglia, such as inhibiting proinflammatory cytokines, promoting phagocytosis, and maintaining the energy metabolism of microglia ${ }^{[60-62]}$. A recent study found that lack of TREM2 amplified TLR4-driven inflammatory responses ${ }^{[63]}$. Moreover, in-vivo studies have demonstrated that TREM2 attenuates neuroinflammation by negatively regulating TLR4-mediated activation of NF-KB signaling ${ }^{[43]}$. In our present study, we found that $\mathrm{DM} / \mathrm{CCH}$ induced activation of the TLR4/NF-KB pathway, and unexpectedly also increased the expression of TREM2. This latter finding is contrary to that of some previous studies ${ }^{[20,64]}$, perhaps due to different models or different observation times. Interestingly, in our present study, curcumin treatment effectively reversed DM/CCH-induced increase in TLR4 and NF-KB levels while further increasing TREM2 expression. Our results in vitro further confirmed these findings. Recently, Takalo et al discovered that phospholipase C gamma 2 (PLCY2) may be associated with TREM2/TLR4 signaling ${ }^{[65]}$. They found that PLCY2 was activated downstream of TREM2 to promote beneficial microglial function, while PLC 2 also acted downstream of the ligand-stimulated TLR pathway to induce inflammatory responses. In the absence of TREM2, microglia are unable to play a protective role in the TREM2/PLCY2 pathway and eventually amplify activation of downstream signaling pathways of TLR. However, whether PLCY2 is associated with curcumin-mediated regulation of TREM2/TLR4 signaling requires further investigation.

Apoptosis plays a major role in neurological diseases, including $A D, P D$, and $\mathrm{VaD}^{[22,66,67]}$. Many signaling pathways regulate apoptosis, including Notch, Bax/Bcl2/caspase3, and IRE1-a/TRAF2/ASK1 pathways ${ }^{[68-70]}$. Among them, $\mathrm{Bax} / \mathrm{Bcl} 2 /$ caspase3 signaling has been extensively studied. Bax is a proapoptotic Bcl2-family protein, while $\mathrm{Bcl} 2$ is an anti-apoptotic protein ${ }^{[71]}$. Additionally, activation of caspase3 is a key event in the execution of DNA fragmentation factor (DFF). The activation of DFF then activates endonucleases, cleaves nuclear DNA, and ultimately leads to cell death. Dysregulation of apoptotic pathways can cause cell death and promote disease progression ${ }^{[72]}$. A recent study found that Bax protein expression was increased while Bcl2 expression was decreased in a mouse model of PD ${ }^{[67]}$. Consistently, our present results showed that expression levels of cleaved-caspase 3 and Bax were significantly increased whereas Bcl2 levels were significantly decreased following $\mathrm{DM} / \mathrm{CCH}$ in vivo, whereas these changes in expression levels were reversed by curcumin treatment. Similar results were 
observed in high-glucose/hypoxia-treated microglia in vitro. Therefore, our findings suggest that curcumin alleviated $\mathrm{DM} / \mathrm{CCH}$-induced and high-glucose/hypoxia-induced pathologies through reducing apoptosis via the $\mathrm{Bax} / \mathrm{Bcl} 2 /$ caspase3 pathway.

Recently, inflammasomes have been reported to be associated with the progression of neurodegenerative disorders $^{[47,73-75]}$. Inflammasomes are large polyprotein complexes that mediate the innate immune response to infectious microorganisms. At present, researchers have found a variety of inflammasomes, such as NLRP1, NLRP3, NLRP6, NLRP7, NLRC4, and AIM2 ${ }^{[76]}$. Among them, the NLRP3 inflammasome is the best characterized and includes a sensor (NLRP3), an adaptor protein (ASC), and an effector protein (caspase1) ${ }^{[77,78]}$. After activation, the NLRP3 inflammasome contributes to the conversion of procaspase 1 into active caspase 1 , resulting in the production of proinflammatory cytokines (e.g., IL-18 and IL-1 $\beta$ ), thereby activating NLRP3 signaling that aggravates neuroinflammation and can promote the progression of neurodegenerative diseases ${ }^{[79,80]}$. Additionally, active caspase 1 can induce pyroptosis via cleavage of GSDMD. As a protein substrate of caspase1, GSDMD has been identified as a key substrate of the inflammasome proteolytic pathway and the main driver of NLRP3-dependent pyroptosis ${ }^{[81]}$. After cleavage of GSDMD, there is an $\mathrm{N}$-terminal cleavage product that remains, which is sufficient to form membranous cores ${ }^{[82]}$. Therefore, pyroptosis is distinct from apoptosis since it is characterized by membrane rupture that causes the release of cytosolic contents to further aggravate inflammatory responses. A recent study has found that NLRP3/caspase1/GSDMD signaling is overactivated in $\mathrm{PD}^{[74]}$. In our present study, we detected the expression of NLRP3-signaling-associated proteins in vivo and in vitro. Our results in vivo showed that curcumin effectively suppressed $\mathrm{DM} / \mathrm{CCH}$-induced overexpression of NLRP3, ASC, cleaved-caspase1, IL-18, and cleavage of GSDMD. Similar results were found in microglia exposed to high glucose combined with hypoxia in vitro. Taken together, these findings suggest that the beneficial effects of curcumin involve modulation on NLRP3-induced pyroptosis. However, the specific mechanism of curcumin suppressing pyroptosis requires further investigation.

\section{Conclusions}

We demonstrated that curcumin attenuated $\mathrm{DM} / \mathrm{CCH}$-induced cognitive deficits and hippocampal neuronal damage in rats, and that its underlying mechanisms were associated with inhibiting neuroinflammation, regulating the TREM2/TLR4/NF-KB pathway, suppressing excessive apoptosis, and mitigating NLRP3-dependent pyroptosis. These findings suggest that curcumin may be useful as a pharmacological strategy for ameliorating $\mathrm{DM} / \mathrm{CCH}$-induced memory deficits.

\section{Abbreviations}

DM Diabetes mellitus

HFD High-fat diet

LFD Low-fat diet 
VaD Vascular dementia

$\mathrm{CCH}$ Chronic cerebral hypoperfusion

BCCAO Bilateral common carotid artery occlusion

TREM2 Triggering receptor expressed on myeloid cells 2

TLR4 Toll-like receptor 4

STZ Streptozotocin

AD Alzheimer's disease

PD Parkinson's disease

\section{Declarations}

\subsection{Ethics approval and consent to participate}

All the animal experiments were approved by the Institutional Animal Care and Use Committee of Shanghai Jiao Tong University.

\subsection{Consent for publication}

Written informed consent for publication was obtained from all participants.

\subsection{Availability of data and materials}

All data generated or analyzed during this work are included in this article.

\subsection{Competing interests}

The authors declare that they have no competing interests

\subsection{Funding}

This study was supported by the Project of National Natural Science Foundation of China (No. 81871103, No.81672243). This study was supported by grants-in-aid from Shanghai Municipal Commission of Science and Technology (Grant No.18DZ1160200)

\subsection{Authors' contributions}

ZYL, ZJW, LY and FJL designed the research. ZYL and ZJW performed the experiments. ZYL, ZJW, ZY and $Z Y X$ analyzed the data. $Z Y L$ and $Z X J$ contributed to the production of the manuscript. All authors read and approved the final manuscript. 


\subsection{Acknowledgements}

We thank Guan Jian for their technical assistance.

\section{References}

1. O'Brien JT, Thomas A. Vascular dementia. Lancet, 2015;386,10004:1698-1706.

2. Duncombe J, Kitamura A, Hase Y, Ihara M, Kalaria RN, Horsburgh K. Chronic cerebral hypoperfusion: a key mechanism leading to vascular cognitive impairment and dementia. Closing the translational gap between rodent models and human vascular cognitive impairment and dementia. Clin Sci (Lond), 2017;131,19:2451-2468.

3. Jing Z, Shi C, Zhu L, Xiang Y, Chen P, Xiong Z, Li W, Ruan Y, Huang L. Chronic cerebral hypoperfusion induces vascular plasticity and hemodynamics but also neuronal degeneration and cognitive impairment. J Cereb Blood Flow Metab, 2015;35,8:1249-1259.

4. Meyer JS, Rauch G, Rauch RA, Haque A. Risk factors for cerebral hypoperfusion, mild cognitive impairment, and dementia. Neurobiol Aging, 2000;21,2:161-169.

5. Nunes Santiago A, Dias Fiuza Ferreira E, Weffort de Oliveira RM, Milani H. Cognitive, neurohistological and mortality outcomes following the four-vessel occlusion/internal carotid artery model of chronic cerebral hypoperfusion: The impact of diabetes and aging. Behav Brain Res, 2018;339,169-178.

6. Zheng Y, Ley SH, Hu FB. Global aetiology and epidemiology of type 2 diabetes mellitus and its complications. Nat Rev Endocrinol, 2018;14,2:88-98.

7. Biessels GJ, Staekenborg S, Brunner E, Brayne C, Scheltens P. Risk of dementia in diabetes mellitus: a systematic review. Lancet Neurol, 2006;5,1:64-74.

8. Biessels GJ, Despa F. Cognitive decline and dementia in diabetes mellitus: mechanisms and clinical implications. Nat Rev Endocrinol, 2018;14,10:591-604.

9. Daulatzai MA. Cerebral hypoperfusion and glucose hypometabolism: Key pathophysiological modulators promote neurodegeneration, cognitive impairment, and Alzheimer's disease. J Neurosci Res, 2017;95,4:943-972.

10. Kwon KJ, Lee EJ, Kim MK, Kim SY, Kim JN, Kim JO, Kim HJ, Kim HY, Han JS, Shin CY, Han SH. Diabetes augments cognitive dysfunction in chronic cerebral hypoperfusion by increasing neuronal cell death: implication of cilostazol for diabetes mellitus-induced dementia. Neurobiol Dis, 2015;73,12-23.

11. Zhang J, Liu Y, Zheng Y, Luo Y, Du Y, Zhao Y, Guan J, Zhang X, Fu J. TREM-2-p38 MAPK signaling regulates neuroinflammation during chronic cerebral hypoperfusion combined with diabetes mellitus. J Neuroinflammation, 2020;17,1:2.

12. Li W, Wei D, Liang J, Xie X, Song K, Huang L. Comprehensive Evaluation of White Matter Damage and Neuron Death and Whole-Transcriptome Analysis of Rats With Chronic Cerebral Hypoperfusion. Front Cell Neurosci, 2019;13,310. 
13. Du SQ, Wang XR, Xiao LY, Tu JF, Zhu W, He T, Liu CZ. Molecular Mechanisms of Vascular Dementia: What Can Be Learned from Animal Models of Chronic Cerebral Hypoperfusion? Mol Neurobiol, 2017;54,5:3670-3682.

14. Belkhelfa M, Beder N, Mouhoub D, Amri M, Hayet R, Tighilt N, Bakheti S, Laimouche S, Azzouz D, Belhadj R, Touil-Boukoffa $\mathrm{C}$. The involvement of neuroinflammation and necroptosis in the hippocampus during vascular dementia. J Neuroimmunol, 2018;320,48-57.

15. Impellizzeri D, Siracusa R, Cordaro M, Crupi R, Peritore AF, Gugliandolo E, D'Amico R, Petrosino S, Evangelista M, Di Paola R, Cuzzocrea S. N-Palmitoylethanolamine-oxazoline (PEA-OXA): A new therapeutic strategy to reduce neuroinflammation, oxidative stress associated to vascular dementia in an experimental model of repeated bilateral common carotid arteries occlusion. Neurobiol Dis, 2019;125,77-91.

16. Ransohoff RM. How neuroinflammation contributes to neurodegeneration. Science, 2016;353,6301:777-783.

17. Perry VH, Holmes C. Microglial priming in neurodegenerative disease. Nat Rev Neurol, 2014;10,4:217224.

18. Feng L, Gao J, Wang Y, Cheong YK, Ren G, Yang Z. Etidronate-zinc Complex Ameliorated Cognitive and Synaptic Plasticity Impairments in 2-Vessel Occlusion Model Rats by Reducing Neuroinflammation. Neuroscience, 2018,390,206-217.

19. Andreasson KI, Bachstetter AD, Colonna M, Ginhoux F, Holmes C, Lamb B, Landreth G, Lee DC, Low D, Lynch MA, Monsonego A, O'Banion MK, Pekny M, Puschmann T, Russek-Blum N, Sandusky LA, Selenica ML, Takata K, Teeling J, Town T, Van Eldik LJ. Targeting innate immunity for neurodegenerative disorders of the central nervous system. J Neurochem, 2016;138,5:653-693.

20. Zhang J, Zheng Y, Luo Y, Du Y, Zhang X, Fu J. Curcumin inhibits LPS-induced neuroinflammation by promoting microglial M2 polarization via TREM2/ TLR4/ NF-KB pathways in BV2 cells. Mol Immunol, 2019;116,29-37.

21. Li W, Yuan H, Yu Y, Cheong YK, Ren G, Yang Z. Etidronate rescues cognitive deficits through improving synaptic transmission and suppressing apoptosis in 2-vessel occlusion model rats. $J$ Neurochem, 2017;140,3:476-484.

22. Sun $M$, Shen $X$, Ma Y. Rehmannioside $A$ attenuates cognitive deficits in rats with vascular dementia (VD) through suppressing oxidative stress, inflammation and apoptosis. Biomed Pharmacother, 2019;120,109492.

23. Wang XX, Zhang B, Xia R, Jia QY. Inflammation, apoptosis and autophagy as critical players in vascular dementia. Eur Rev Med Pharmacol Sci, 2020;24,18:9601-9614.

24. Zhang G, Liu A, Zhou Y, San X, Jin T, Jin Y. Panax ginseng ginsenoside-Rg2 protects memory impairment via anti-apoptosis in a rat model with vascular dementia. J Ethnopharmacol, 2008;115,3:441-448.

25. Liu XL, Lu J, Xing J. Stabilization of HIF-1 a modulates VEGF and Caspase-3 in the hippocampus of rats following transient global ischemia induced by asphyxial cardiac arrest. Life Sci, 2016;151,243- 
249.

26. Zhao N, Sun C, Zheng M, Liu S, Shi R. Amentoflavone suppresses amyloid $\beta 1-42$ neurotoxicity in Alzheimer's disease through the inhibition of pyroptosis. Life Sci, 2019;239,117043.

27. Li Y, Xu P, Shan J, Sun W, Ji X, Chi T, Liu P, Zou L. Interaction between hyperphosphorylated tau and pyroptosis in forskolin and streptozotocin induced AD models. Biomed Pharmacother, 2020;121,109618.

28. Schroder K, Tschopp J. The inflammasomes. Cell, 2010;140,6:821-832.

29. He Y, Hara H, Núñez G. Mechanism and Regulation of NLRP3 Inflammasome Activation. Trends Biochem Sci, 2016;41,12:1012-1021.

30. Wree A, Eguchi A, McGeough MD, Pena CA, Johnson CD, Canbay A, Hoffman HM, Feldstein AE. NLRP3 inflammasome activation results in hepatocyte pyroptosis, liver inflammation, and fibrosis in mice. Hepatology, 2014;59,3:898-910.

31. Kahkhaie KR, Mirhosseini A, Aliabadi A, Mohammadi A, Mousavi MJ, Haftcheshmeh SM, Sathyapalan T, Sahebkar A. Curcumin: a modulator of inflammatory signaling pathways in the immune system. Inflammopharmacology, 2019;27,5:885-900.

32. Hussain Z, Thu HE, Amjad MW, Hussain F, Ahmed TA, Khan S. Exploring recent developments to improve antioxidant, anti-inflammatory and antimicrobial efficacy of curcumin: A review of new trends and future perspectives. Mater Sci Eng C Mater Biol Appl, 2017;77,1316-1326.

33. Ghanaatian N, Lashgari NA, Abdolghaffari AH, Rajaee SM, Panahi Y, Barreto GE, Butler AE, Sahebkar A. Curcumin as a therapeutic candidate for multiple sclerosis: Molecular mechanisms and targets. $J$ Cell Physiol, 2019;234,8:12237-12248.

34. Reddy PH, Manczak M, Yin X, Grady MC, Mitchell A, Kandimalla R, Kuruva CS. Protective effects of a natural product, curcumin, against amyloid $\beta$ induced mitochondrial and synaptic toxicities in Alzheimer's disease. J Investig Med, 2016;64,8:1220-1234.

35. Yu SY, Zhang M, Luo J, Zhang L, Shao Y, Li G. Curcumin ameliorates memory deficits via neuronal nitric oxide synthase in aged mice. Prog Neuropsychopharmacol Biol Psychiatry, 2013;45,47-53.

36. Ghasemi F, Bagheri H, Barreto GE, Read MI, Sahebkar A. Effects of Curcumin on Microglial Cells. Neurotox Res, 2019;36,1:12-26.

37. Li Y, Zhang T, Zhang X, Zou W, Gong X, Fu J. Cinepazide Maleate Improves Cognitive Function and Protects Hippocampal Neurons in Diabetic Rats with Chronic Cerebral Hypoperfusion. Biol Pharm Bull, 2017;40,3:249-255.

38. Zou W, Song Y, Li Y, Du Y, Zhang X, Fu J. The Role of Autophagy in the Correlation Between Neuron Damage and Cognitive Impairment in Rat Chronic Cerebral Hypoperfusion. Mol Neurobiol, 2018;55,1:776-791.

39. Guo T, Fang J, Tong ZY, He S, Luo Y. Transcranial Direct Current Stimulation Ameliorates Cognitive Impairment via Modulating Oxidative Stress, Inflammation, and Autophagy in a Rat Model of Vascular Dementia. Front Neurosci, 2020;14,28. 
40. Cervellati C, Trentini A, Pecorelli A, Valacchi G. Inflammation in Neurological Disorders: The Thin Boundary Between Brain and Periphery. Antioxid Redox Signal, 2020;33,3:191-210.

41. Wang L, Yang JW, Lin LT, Huang J, Wang XR, Su XT, Cao Y, Fisher M, Liu CZ. Acupuncture Attenuates Inflammation in Microglia of Vascular Dementia Rats by Inhibiting miR-93-Mediated TLR4/MyD88/NF-kB Signaling Pathway. Oxid Med Cell Longev, 2020;2020,8253904.

42. Long $\mathrm{H}$, Zhong G, Wang $\mathrm{C}$, Zhang J, Zhang Y, Luo J, Shi S. TREM2 Attenuates Aß1-42-Mediated Neuroinflammation in BV-2 Cells by Downregulating TLR Signaling. Neurochem Res, 2019;44,8:18301839.

43. Ren M, Guo Y, Wei X, Yan S, Qin Y, Zhang X, Jiang F, Lou H. TREM2 overexpression attenuates neuroinflammation and protects dopaminergic neurons in experimental models of Parkinson's disease. Exp Neurol, 2018;302,205-213.

44. Liu T, Zhang L, Joo D, Sun SC. NF-KB signaling in inflammation. Signal Transduct Target Ther, 2017;2,17023-.

45. Zhao T, Fu Y, Sun H, Liu X. Ligustrazine suppresses neuron apoptosis via the Bax/Bcl-2 and caspase3 pathway in PC12 cells and in rats with vascular dementia. IUBMB Life, 2018;70,1:60-70.

46. Zhu W, Wang XR, Du SQ, Yan CQ, Yang NN, Lin LL, Shi GX, Liu CZ. Anti-oxidative and Anti-apoptotic Effects of Acupuncture: Role of Thioredoxin-1 in the Hippocampus of Vascular Dementia Rats. Neuroscience, 2018;379,281-291.

47. Wang S, Yuan YH, Chen NH, Wang HB. The mechanisms of NLRP3 inflammasome/pyroptosis activation and their role in Parkinson's disease. Int Immunopharmacol, 2019;67,458-464.

48. Paldino E, D'Angelo V, Laurenti D, Angeloni C, Sancesario G, Fusco FR. Modulation of Inflammasome and Pyroptosis by Olaparib, a PARP-1 Inhibitor, in the R6/2 Mouse Model of Huntington's Disease. Cells, 2020;9,10:

49. Su SH, Wu YF, Lin Q, Wang DP, Hai J. URB597 protects against NLRP3 inflammasome activation by inhibiting autophagy dysfunction in a rat model of chronic cerebral hypoperfusion. $J$ Neuroinflammation, 2019;16,1:260.

50. Choi BR, Kim DH, Back DB, Kang CH, Moon WJ, Han JS, Choi DH, Kwon KJ, Shin CY, Kim BR, Lee J, Han SH, Kim HY. Characterization of White Matter Injury in a Rat Model of Chronic Cerebral Hypoperfusion. Stroke, 2016;47,2:542-547.

51. Yan W, Pang M, Yu Y, Gou X, Si P, Zhawatibai A, Zhang Y, Zhang M, Guo T, Yi X, Chen L. The neuroprotection of liraglutide on diabetic cognitive deficits is associated with improved hippocampal synapses and inhibited neuronal apoptosis. Life Sci, 2019;231,116566.

52. Bang J, Kim MS, Jeon WK. Mumefural Ameliorates Cognitive Impairment in Chronic Cerebral Hypoperfusion via Regulating the Septohippocampal Cholinergic System and Neuroinflammation. Nutrients, 2019;11,11:

53. Han B, Jiang W, Liu H, Wang J, Zheng K, Cui P, Feng Y, Dang C, Bu Y, Wang QM, Ju Z, Hao J. Upregulation of neuronal PGC-1a ameliorates cognitive impairment induced by chronic cerebral hypoperfusion. Theranostics, 2020;10,6:2832-2848. 
54. Miyanohara J, Kakae M, Nagayasu K, Nakagawa T, Mori Y, Arai K, Shirakawa H, Kaneko S. TRPM2 Channel Aggravates CNS Inflammation and Cognitive Impairment via Activation of Microglia in Chronic Cerebral Hypoperfusion. J Neurosci, 2018;38,14:3520-3533.

55. Hernández C, Bogdanov P, Gómez-Guerrero C, Sampedro J, Solà-Adell C, Espejo C, García-Ramírez M, Prieto I, Egido J, Simó R. SOCS1-Derived Peptide Administered by Eye Drops Prevents Retinal Neuroinflammation and Vascular Leakage in Experimental Diabetes. Int J Mol Sci, 2019;20,15:

56. Azul L, Leandro A, Boroumand P, Klip A, Seiça R, Sena CM. Increased inflammation, oxidative stress and a reduction in antioxidant defense enzymes in perivascular adipose tissue contribute to vascular dysfunction in type 2 diabetes. Free Radic Biol Med, 2020;146,264-274.

57. Shi H, Wang XL, Quan HF, Yan L, Pei XY, Wang R, Peng XD. Effects of Betaine on LPS-Stimulated Activation of Microglial M1/M2 Phenotypes by Suppressing TLR4/NF-KB Pathways in N9 Cells. Molecules, 2019;24,2:

58. Zhou J, Yu W, Zhang M, Tian X, Li Y, Lü Y. Imbalance of Microglial TLR4/TREM2 in LPS-Treated APP/PS1 Transgenic Mice: A Potential Link Between Alzheimer's Disease and Systemic Inflammation. Neurochem Res, 2019;44,5:1138-1151.

59. Rosciszewski G, Cadena V, Murta V, Lukin J, Villarreal A, Roger T, Ramos AJ. Toll-Like Receptor 4 (TLR4) and Triggering Receptor Expressed on Myeloid Cells-2 (TREM-2) Activation Balance Astrocyte Polarization into a Proinflammatory Phenotype. Mol Neurobiol, 2018;55,5:3875-3888.

60. Takahashi K, Rochford CD, Neumann H. Clearance of apoptotic neurons without inflammation by microglial triggering receptor expressed on myeloid cells-2. J Exp Med, 2005;201,4:647-657.

61. Ulland TK, Song WM, Huang SC, Ulrich JD, Sergushichev A, Beatty WL, Loboda AA, Zhou Y, Cairns NJ, Kambal A, Loginicheva E, Gilfillan S, Cella M, Virgin HW, Unanue ER, Wang Y, Artyomov MN, Holtzman DM, Colonna M. TREM2 Maintains Microglial Metabolic Fitness in Alzheimer's Disease. Cell, 2017;170,4:649-663.e613.

62. Kleinberger G, Yamanishi Y, Suárez-Calvet M, Czirr E, Lohmann E, Cuyvers E, Struyfs H, Pettkus N, Wenninger-Weinzierl A, Mazaheri F, Tahirovic S, Lleó A, Alcolea D, Fortea J, Willem M, Lammich S, Molinuevo JL, Sánchez-Valle R, Antonell A, Ramirez A, Heneka MT, Sleegers K, van der Zee J, Martin JJ, Engelborghs S, Demirtas-Tatlidede A, Zetterberg H, Van Broeckhoven C, Gurvit H, Wyss-Coray T, Hardy J, Colonna M, Haass C. TREM2 mutations implicated in neurodegeneration impair cell surface transport and phagocytosis. Sci Trans/ Med, 2014;6,243:243ra286.

63. Perugorria MJ, Esparza-Baquer A, Oakley F, Labiano I, Korosec A, Jais A, Mann J, Tiniakos D, SantosLaso A, Arbelaiz A, Gawish R, Sampedro A, Fontanellas A, Hijona E, Jimenez-Agüero R, Esterbauer H, Stoiber D, Bujanda L, Banales JM, Knapp S, Sharif O, Mann DA. Non-parenchymal TREM-2 protects the liver from immune-mediated hepatocellular damage. Gut, 2019;68,3:533-546.

64. Cheng-Hathaway PJ, Reed-Geaghan EG, Jay TR, Casali BT, Bemiller SM, Puntambekar SS, von Saucken VE, Williams RY, Karlo JC, Moutinho M, Xu G, Ransohoff RM, Lamb BT, Landreth GE. The Trem2 R47H variant confers loss-of-function-like phenotypes in Alzheimer's disease. Mol Neurodegener, 2018;13,1:29. 
65. Takalo M, Wittrahm R, Wefers B, Parhizkar S, Jokivarsi K, Kuulasmaa T, Mäkinen P, Martiskainen H, Wurst W, Xiang X, Marttinen M, Poutiainen P, Haapasalo A, Hiltunen M, Haass C. The Alzheimer's disease-associated protective Plcy2-P522R variant promotes immune functions. Mol Neurodegener, 2020;15,1:52.

66. Bamberger ME, Landreth GE. Inflammation, apoptosis, and Alzheimer's disease. Neuroscientist, 2002;8,3:276-283.

67. Liu J, Liu W, Lu Y, Tian H, Duan C, Lu L, Gao G, Wu X, Wang X, Yang H. Piperlongumine restores the balance of autophagy and apoptosis by increasing BCL2 phosphorylation in rotenone-induced Parkinson disease models. Autophagy, 2018;14,5:845-861.

68. Zhang J, Liang Y, Lin Y, Liu Y, YouYou, Yin W. IRE1a-TRAF2-ASK1 pathway is involved in CSTMPinduced apoptosis and ER stress in human non-small cell lung cancer A549 cells. Biomed Pharmacother, 2016;82,281-289.

69. Lin CH, Wu MR, Li CH, Cheng HW, Huang SH, Tsai CH, Lin FL, Ho JD, Kang JJ, Hsiao G, Cheng YW. Editor's Highlight: Periodic Exposure to Smartphone-Mimic Low-Luminance Blue Light Induces Retina Damage Through Bcl-2/BAX-Dependent Apoptosis. Toxicol Sci, 2017;157,1:196-210.

70. Jin Z, Guo P, Li X, Ke J, Wang Y, Wu H. Neuroprotective effects of irisin against cerebral ischemia/ reperfusion injury via Notch signaling pathway. Biomed Pharmacother, 2019;120,109452.

71. Liao LX, Zhao MB, Dong X, Jiang Y, Zeng KW, Tu PF. TDB protects vascular endothelial cells against oxygen-glucose deprivation/reperfusion-induced injury by targeting miR-34a to increase Bcl-2 expression. Sci Rep, 2016;6,37959.

72. Elmore S. Apoptosis: a review of programmed cell death. Toxicol Pathol, 2007;35,4:495-516.

73. Feng YS, Tan ZX, Wu LY, Dong F, Zhang F. The involvement of NLRP3 inflammasome in the treatment of Alzheimer's disease. Ageing Res Rev, 2020;64,101192.

74. Rui W, Li S, Xiao H, Xiao M, Shi J. Baicalein Attenuates Neuroinflammation by Inhibiting NLRP3/caspase-1/GSDMD Pathway in MPTP Induced Mice Model of Parkinson's Disease. Int J Neuropsychopharmacol, 2020;

75. Shao BZ, Cao Q, Liu C. Targeting NLRP3 Inflammasome in the Treatment of CNS Diseases. Front Mol Neurosci, 2018;11,320.

76. Strowig T, Henao-Mejia J, Elinav E, Flavell R. Inflammasomes in health and disease. Nature, 2012;481,7381:278-286.

77. Kanneganti TD, Lamkanfi M, Núñez G. Intracellular NOD-like receptors in host defense and disease. Immunity, 2007;27,4:549-559.

78. Thawkar BS, Kaur G. Inhibitors of NF-KB and P2X7/NLRP3/Caspase 1 pathway in microglia: Novel therapeutic opportunities in neuroinflammation induced early-stage Alzheimer's disease. $J$ Neuroimmunol, 2019;326,62-74.

79. Swaroop S, Mahadevan A, Shankar SK, Adlakha YK, Basu A. HSP60 critically regulates endogenous IL-1 $\beta$ production in activated microglia by stimulating NLRP3 inflammasome pathway. $J$ Neuroinflammation, 2018;15,1:177. 
80. Swanson KV, Deng M, Ting JP. The NLRP3 inflammasome: molecular activation and regulation to therapeutics. Nat Rev Immunol, 2019;19,8:477-489.

81. Shi J, Zhao Y, Wang K, Shi X, Wang Y, Huang H, Zhuang Y, Cai T, Wang F, Shao F. Cleavage of GSDMD by inflammatory caspases determines pyroptotic cell death. Nature, 2015;526,7575:660-665.

82. Liu X, Zhang Z, Ruan J, Pan Y, Magupalli VG, Wu H, Lieberman J. Inflammasome-activated gasdermin D causes pyroptosis by forming membrane pores. Nature, 2016;535,7610:153-158.

\section{Table}

Table 1. Primers for qPCR

\begin{tabular}{|c|c|c|}
\hline Gene & Sense $\left(5^{\prime}-3\right)$ & Anti-sense $\left(5^{\prime}-3\right)$ \\
\hline \multicolumn{3}{|l|}{ Mouse } \\
\hline IL-1 $1 \beta$ & TTTGAAGTTGACGGACCCCAA & CACAGCTTCTCCACAGCCACA \\
\hline IL-6 & CTCCCAACAGACCTGTCTATAC & CCATTGCACAACTCTTTTCTCA \\
\hline TNF- $\alpha$ & ATGTCTCAGCCTCTTCTCATTC & GCTTGTCACTCGAATTTTGAGA \\
\hline iNOS & GGGCTGTCACGGAGATCAATG & GCCCGGTACTCATTCTGCATG \\
\hline $\operatorname{cox} 2$ & GGGCCATGGAGTGGACTTAAA & TGCAGGTTCTCAGGGATGTG \\
\hline Arg-1 & CATATCTGCCAAAGACATCGTG & GACATCAAAGCTCAGGTGAATC \\
\hline TREM2 & TCATGTACTTATGACGCCTTGATTGCCCTCTTC & GAGGTTCTTCAGAGTGATGGTGCCATGATG \\
\hline TLR4 & TACTTTGCTAG & GTTCTGATCAGCTC \\
\hline Bax & TTGCTACAGGGTTTCATCCAGG & GCAAAGTAGAAGAGGGCAACCA \\
\hline Bcl2 & CTACCGTCGTGACTTCGCAGA & ACACATGACCCCACCGAAC \\
\hline caspase3 & GTCTGACTGGAAAGCCGAAAC & GACTGGATGAACCACGACCC \\
\hline NLRP3 & GCCGTCTACGTCTTCTTCCTTTCC & CATCCGCAGCCAGTGAACAGAG \\
\hline IL-18 & AGACCTGGAATCAGACAACTTT & TCAGTCATATCCTCGAACACAG \\
\hline actin & CTACCTCATGAAGATCCTGACC & CACAGCTTCTCTTTGATGTCAC \\
\hline \multicolumn{3}{|l|}{ Rat } \\
\hline IL-1 $\beta$ & TGACCTGTTCTTTGAGGCTGAC & CATCATCCCACGAGTCACAGAG \\
\hline IL -6 & AATGAGAAAAGAGTTGTGCAATGG & AGGTAGAAACGGAACTCCAGAAA \\
\hline TNF- $\alpha$ & CCACCACGCTCTTCTGTCTACTG & TGGGCTACGGGCTTGTCACT \\
\hline Arg-1 & CTTTCTCTAAGGGACAGCCTC & ATCCACAAAGGCCAGATCCC \\
\hline IL-4 & GCTGTCACCCTGTTCTGCTTT & AATTTGCGAAGCACCCTGGAA \\
\hline IL-10 & TGGTAGAAGTGATGCCCCAGG & AAATCGATGACAGCGTCGCAG \\
\hline TREM2 & GCTGGAACTGTCACCATCACTCTG & AGCACCTCCACCACGACCTTC \\
\hline TLR4 & TTGCTGCCAACATCATCCAGGAAG & CAGAGCGGCTACTCAGAAACTGC \\
\hline $\operatorname{Bax}$ & TTGCAGACGGCAACTTCAACTG & CTTTAGTGCACAGGGCCTTGAG \\
\hline $\mathrm{Bcl} 2$ & TCTTTGAGTTCGGTGGGGTCA & AGTTCCACAAAGGCATCCCAG \\
\hline caspase3 & ACAGAGCTGGACTGCGGTATT & TACAGTTTCAGCATGGCGCAA \\
\hline NLRP3 & GCAGCGATCAACAGGCGAGAC & TCCCAGCAAACCTATCCACTCCTC \\
\hline IL-18 & CGACCGAACAGCCAACGAATCC & TCACAGATAGGGTCACAGCCAGTC \\
\hline actin & CGTTGACATCCGTAAAGACCTC & TAGGAGCCAGGGCAGTAATCT \\
\hline
\end{tabular}

\section{Figures}


A

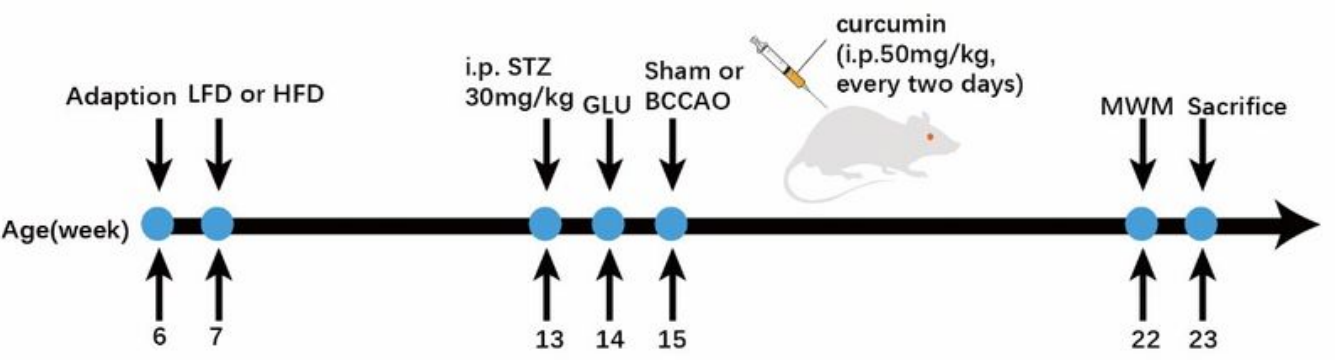

B

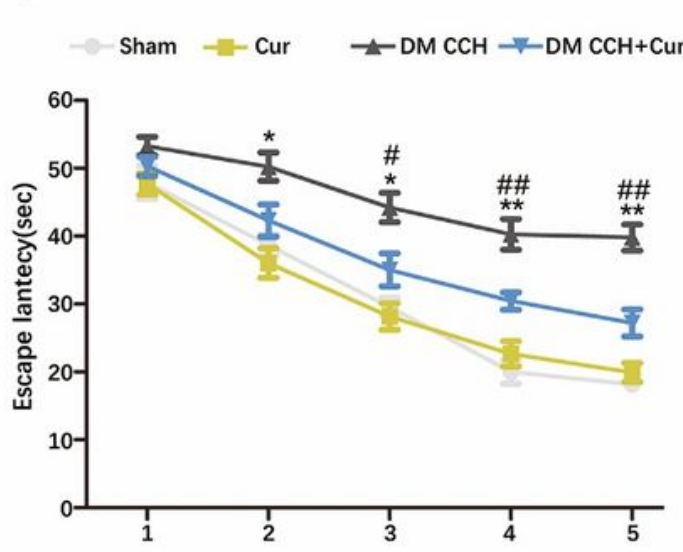

C

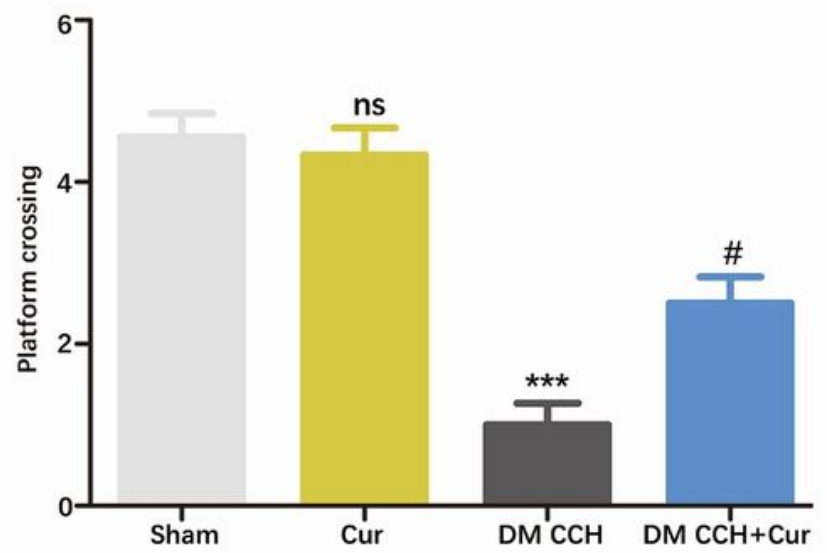

D

\section{E}
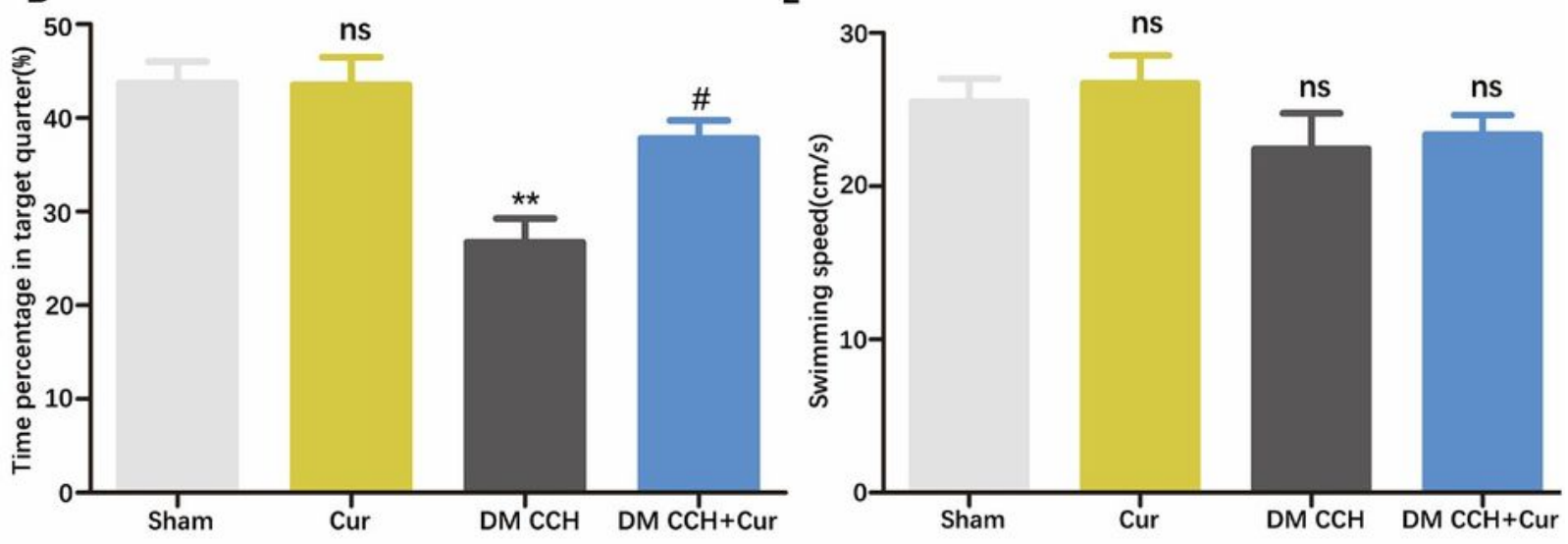

$\mathrm{F}$
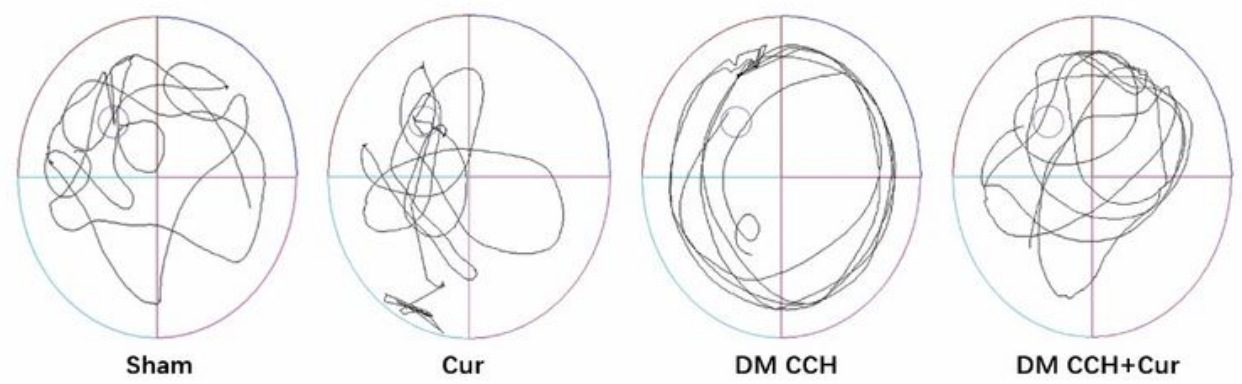

Figure 1

Curcumin alleviates $\mathrm{DM} / \mathrm{CCH}$-induced impairments in spatial learning and memory. A Schematic diagram of the experimental scheme of the MWM. B Escape latencies of rats in the hidden platform task. C The number of crossings across the previous location of the platform in the probe test. D Time spent in the target quadrant during the probe trial. E Swimming speed in the probe trial. F Trajectories of rats in the 
MWM. Values are expressed as the mean \pm SEM $(n=9 / 8) .{ }^{*} P<0.05 ;{ }^{\star *} P<0.01, \star \star \star P<0.001$ vs. Sham; \#P $<0.05 ; \#$ \# $<0.01$ DM/CCH vs. DM/CCH + Cur. Cur, Curcumin

A

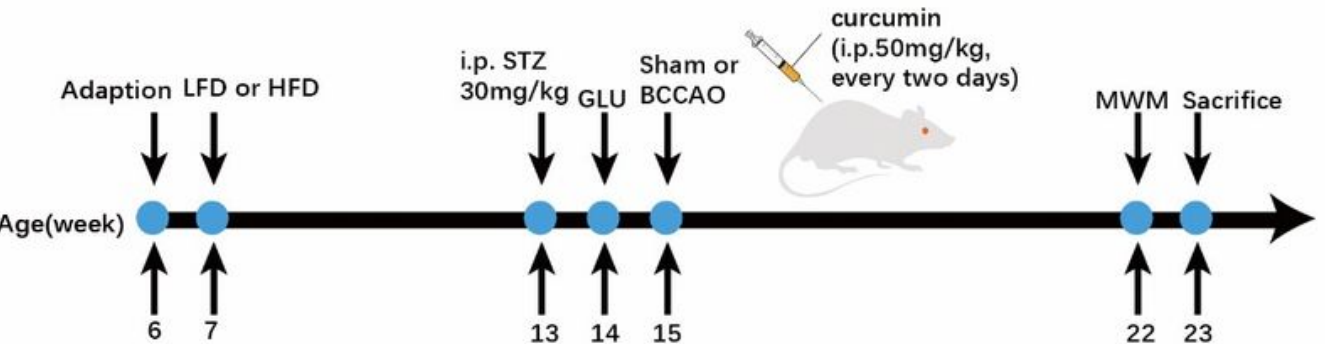

B

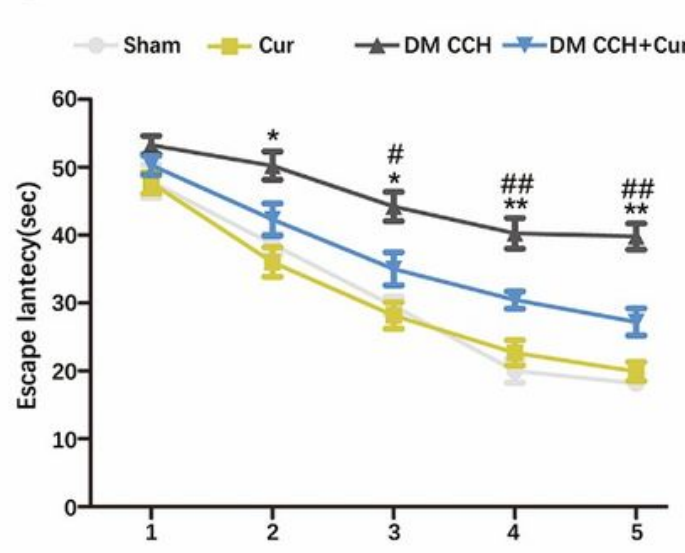

C

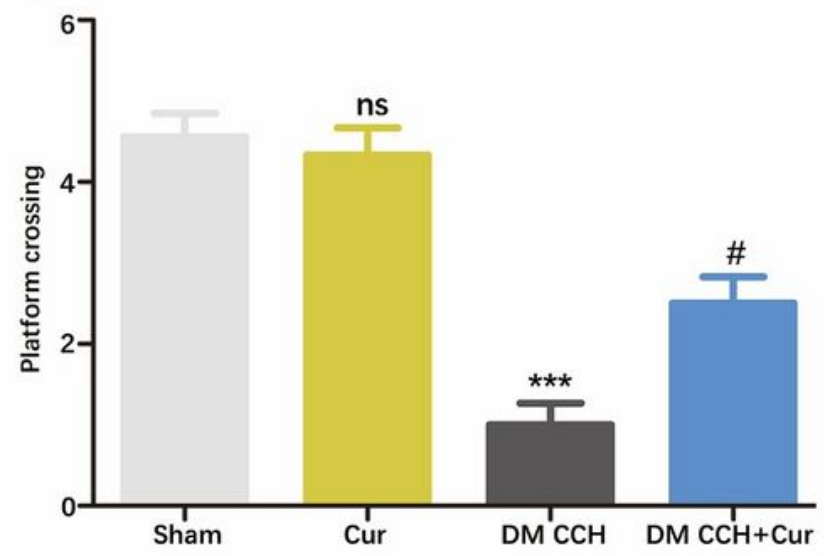

D

$E$
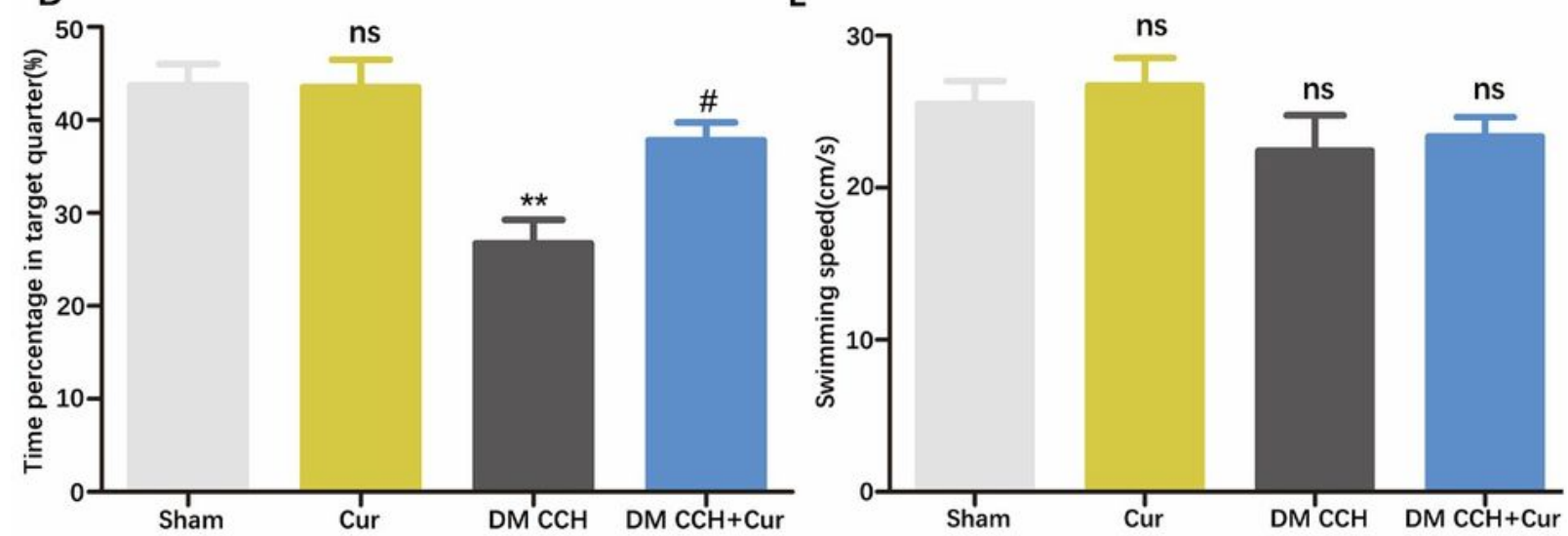

$\mathrm{F}$
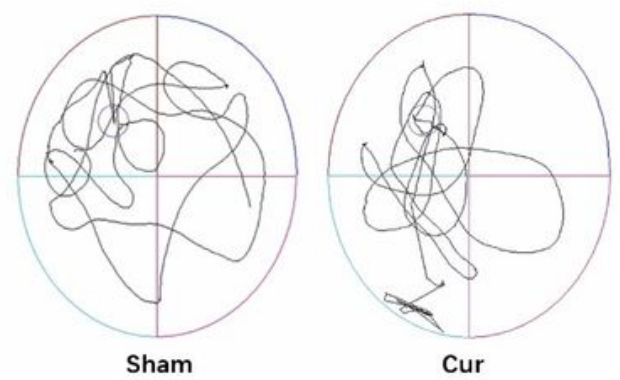

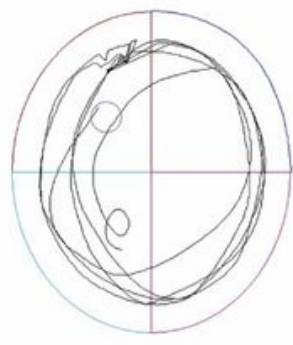

$\mathrm{DM} \mathrm{CCH}$

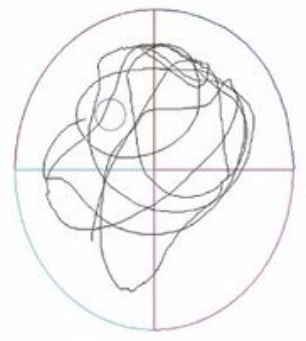

$\mathrm{DM} \mathrm{CCH}+\mathrm{Cur}$

Figure 1

Curcumin alleviates DM/CCH-induced impairments in spatial learning and memory. A Schematic diagram of the experimental scheme of the MWM. B Escape latencies of rats in the hidden platform task. C The number of crossings across the previous location of the platform in the probe test. D Time spent in the 
target quadrant during the probe trial. E Swimming speed in the probe trial. F Trajectories of rats in the MWM. Values are expressed as the mean \pm SEM $(n=9 / 8)$. ${ }^{\star} P<0.05 ; * \star P<0.01, * \star * P<0.001$ vs. Sham; $\# \mathrm{P}<0.05 ; \# \# \mathrm{P}<0.01 \mathrm{DM} / \mathrm{CCH}$ vs. DM/CCH + Cur. Cur, Curcumin
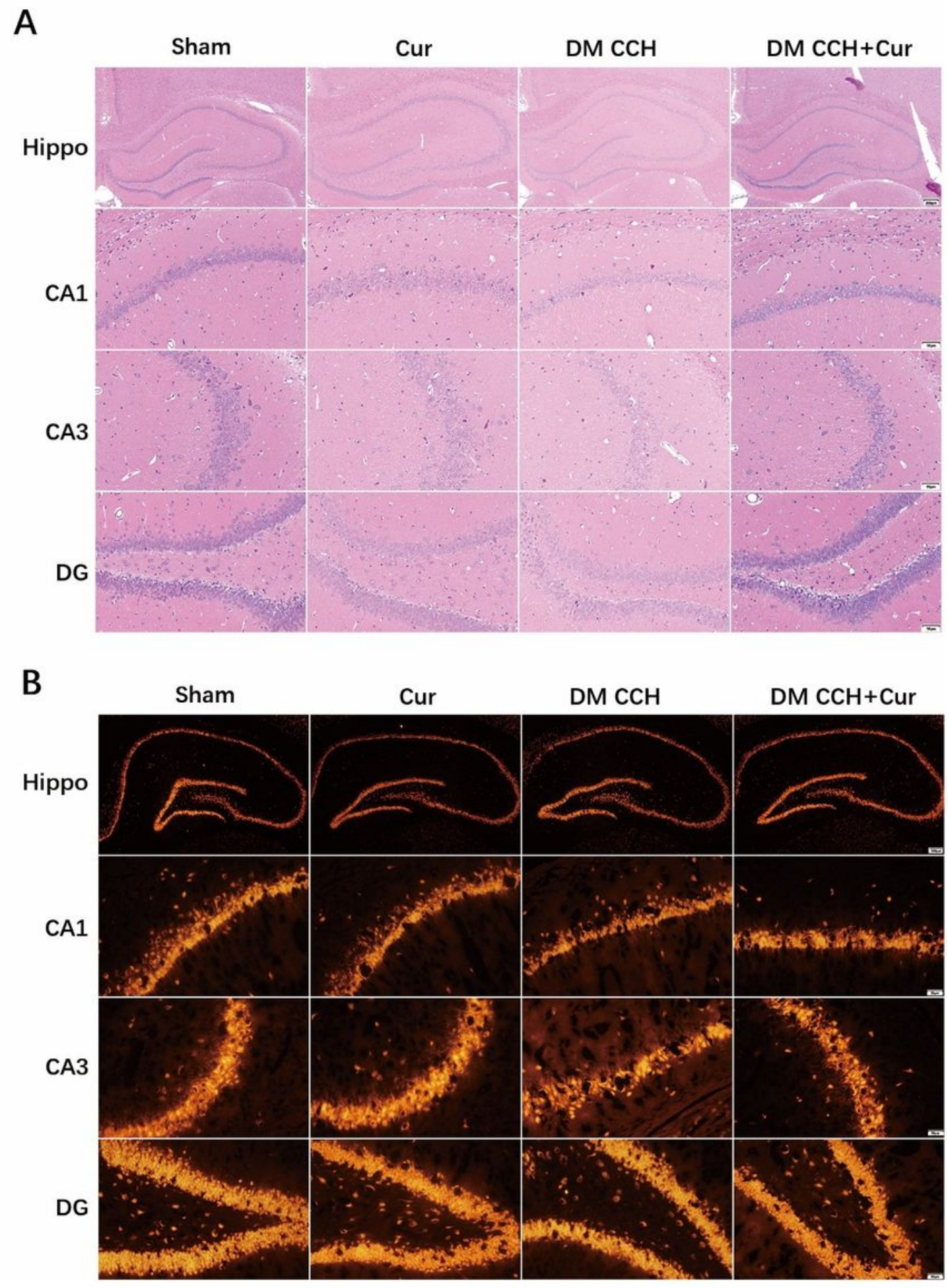

Figure 2

Curcumin attenuates neuronal death in $\mathrm{DM} / \mathrm{CCH}$ rats. A, B Hippocampal sections of the CA1, CA3, and DG regions were obtained and stained with HE and NeuN (magnification 40x or 200x, scale bar $=200 \mu \mathrm{m}$ 
or $50 \mu \mathrm{m})$. Hippo, hippocampus; Cur, Curcumin
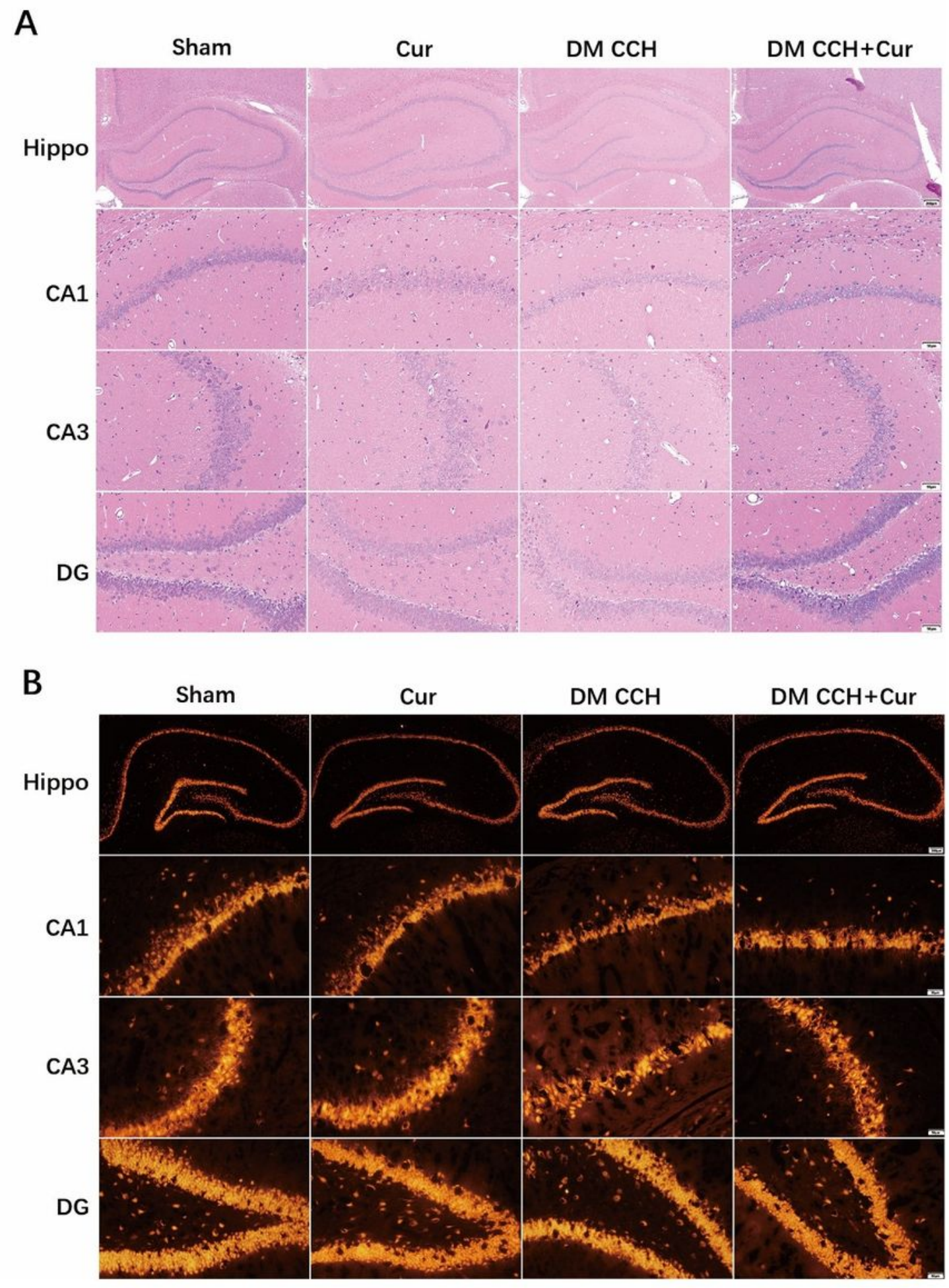

Figure 2

Curcumin attenuates neuronal death in $\mathrm{DM} / \mathrm{CCH}$ rats. A, B Hippocampal sections of the CA1, CA3, and DG regions were obtained and stained with HE and NeuN (magnification 40x or 200x, scale bar $=200 \mu \mathrm{m}$ or $50 \mu \mathrm{m})$. Hippo, hippocampus; Cur, Curcumin 
A
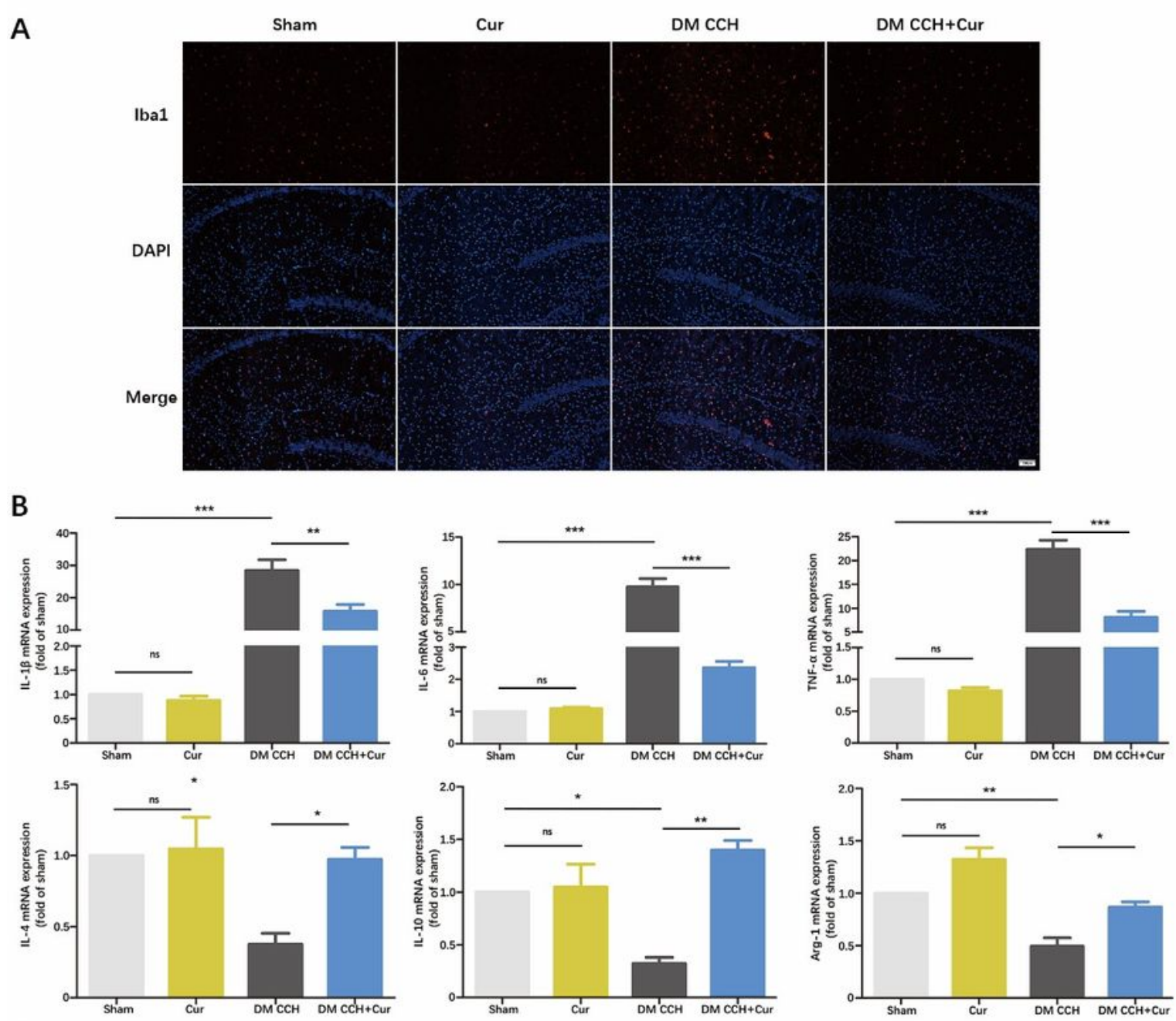

C
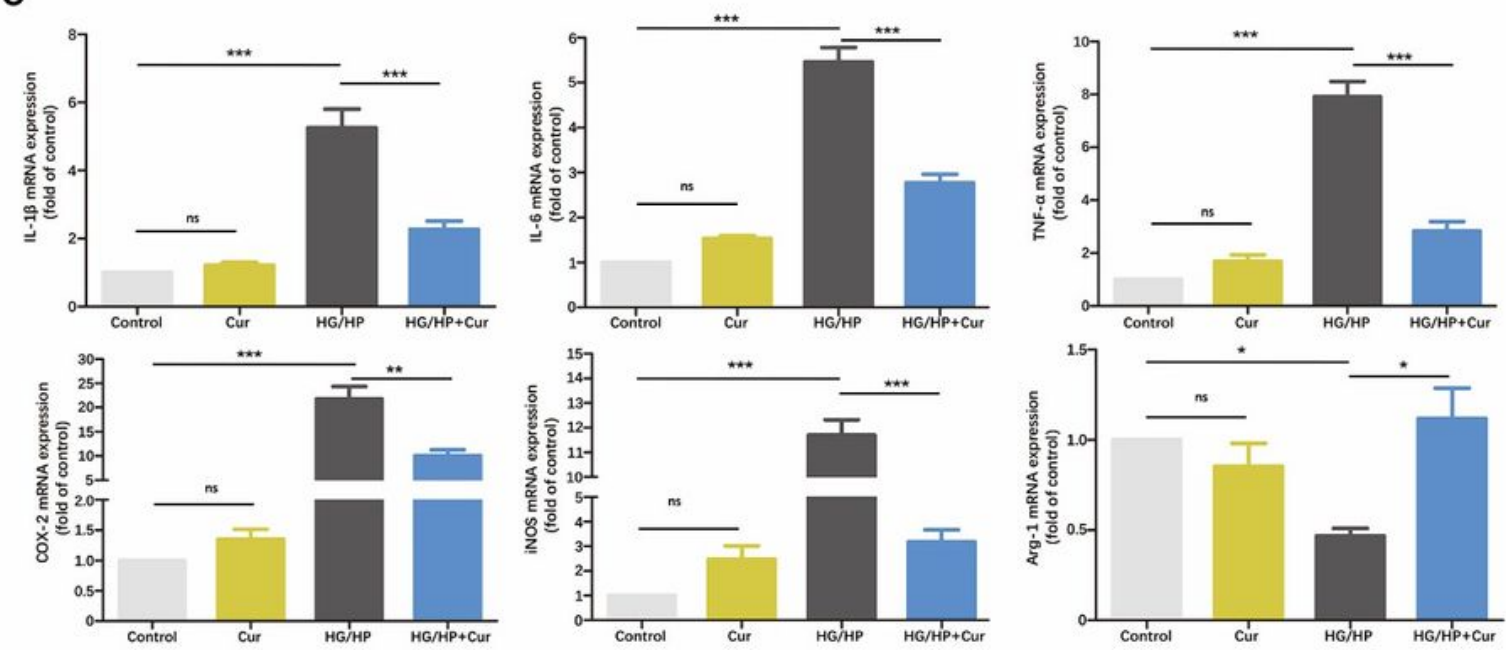

\section{Figure 3}

Curcumin inhibits $\mathrm{DM} / \mathrm{CCH}$-induced neuroinflammation in the rat hippocampus. A. Representative micrographs showing microglial activation in the CA1 region, using an anti-ionized calcium-binding adaptor protein 1 (Iba1) antibody (red) (magnification 100x, scale bar =100 $\mu \mathrm{m}$ ). B, C mRNA levels of proinflammatory cytokines (IL-1 $\beta$, IL-6, TNF-a, iNOS, and COX2) and anti-inflammatory cytokines (IL-4, IL-10, and Arg-1) were detected by qRT-PCR. Cur, Curcumin 
A
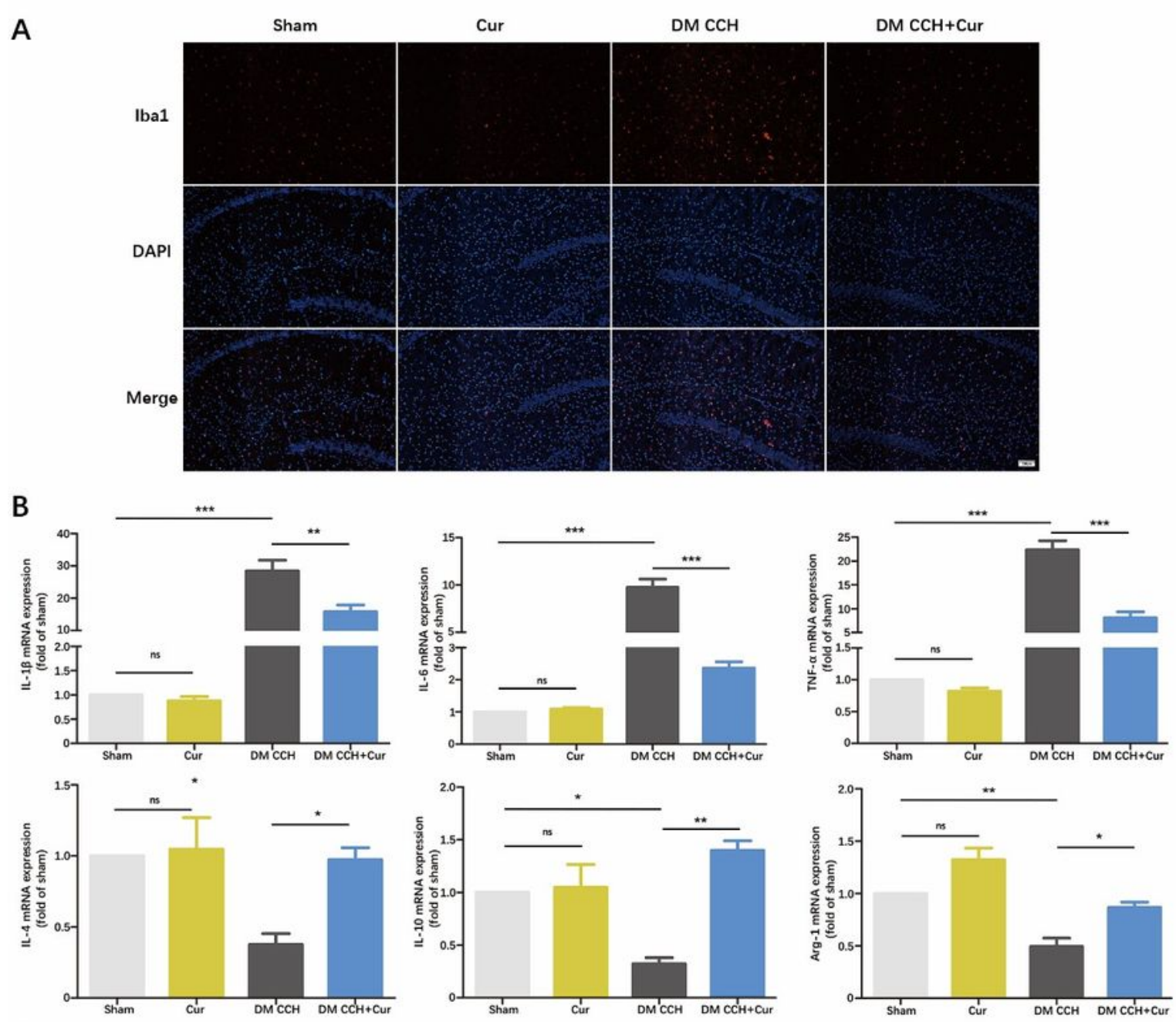

C
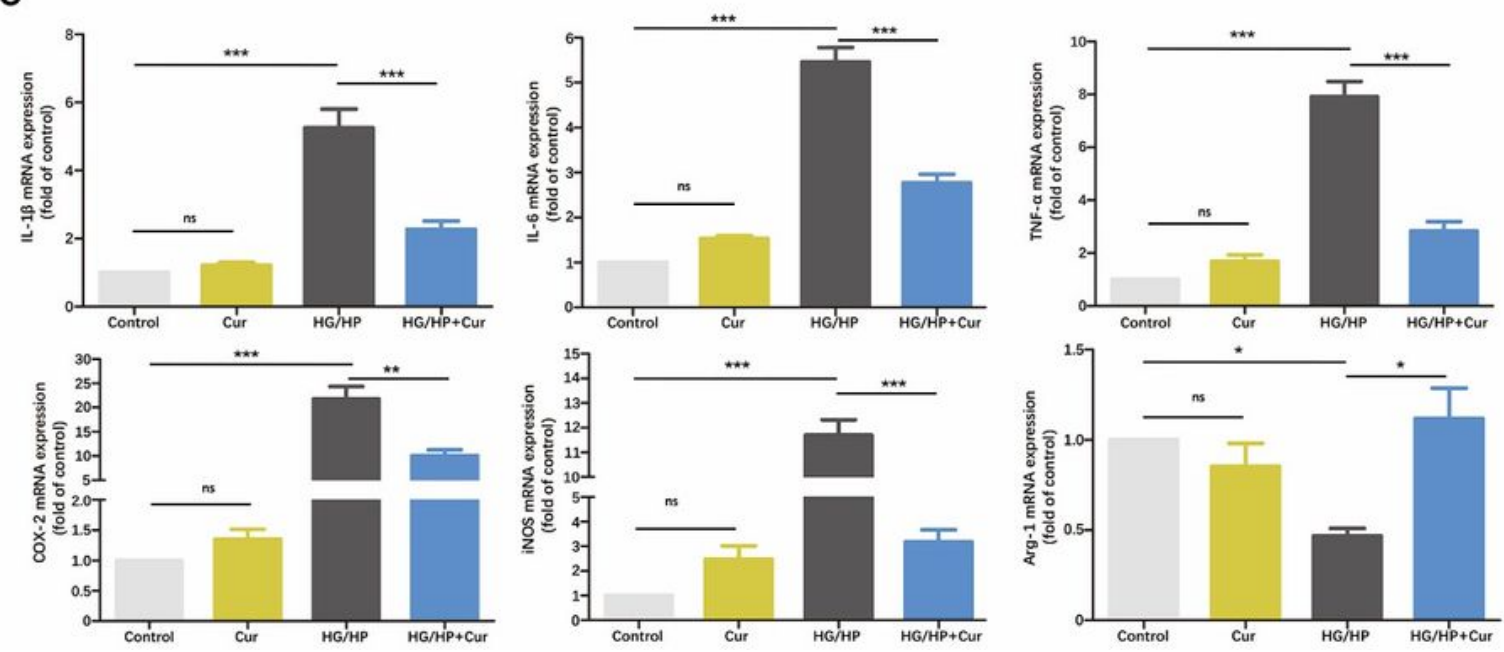

\section{Figure 3}

Curcumin inhibits $\mathrm{DM} / \mathrm{CCH}$-induced neuroinflammation in the rat hippocampus. A. Representative micrographs showing microglial activation in the CA1 region, using an anti-ionized calcium-binding adaptor protein 1 (Iba1) antibody (red) (magnification 100x, scale bar =100 $\mu \mathrm{m}$ ). B, C mRNA levels of proinflammatory cytokines (IL-1 $\beta$, IL-6, TNF-a, iNOS, and COX2) and anti-inflammatory cytokines (IL-4, IL-10, and Arg-1) were detected by qRT-PCR. Cur, Curcumin 
A

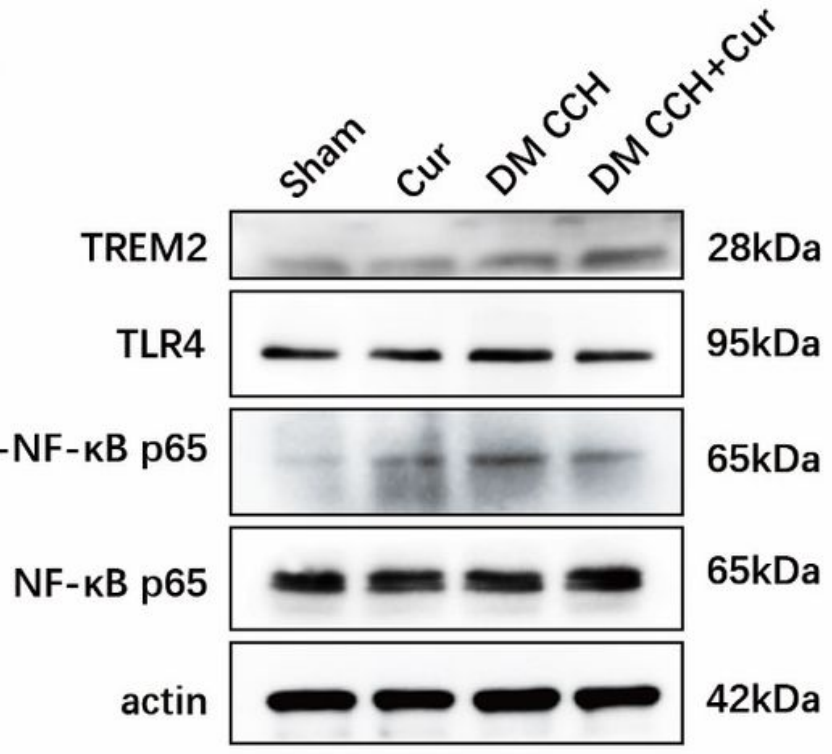

B
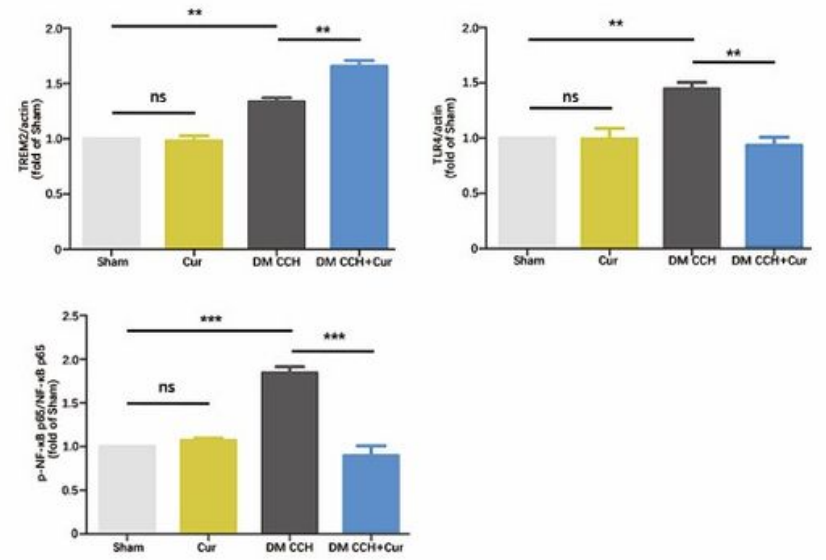

$E$

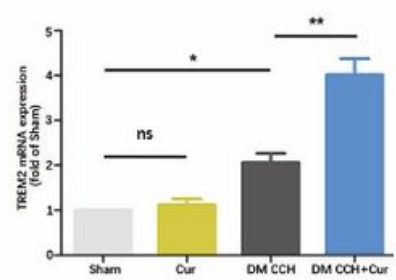

C

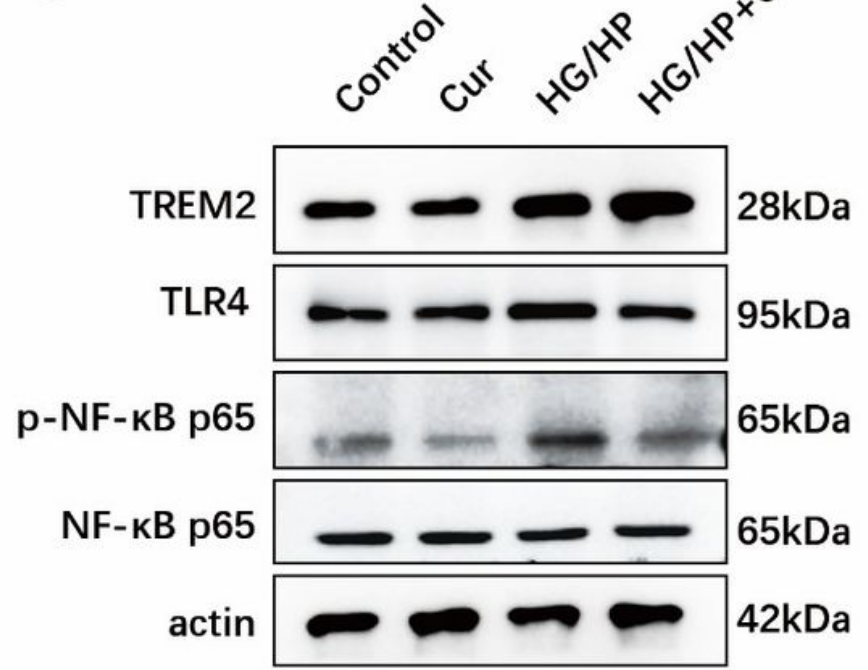

D
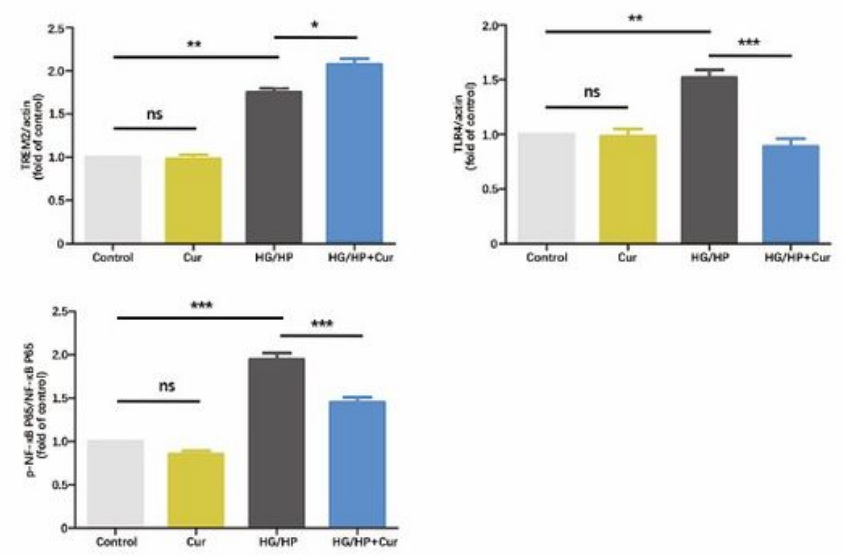

F

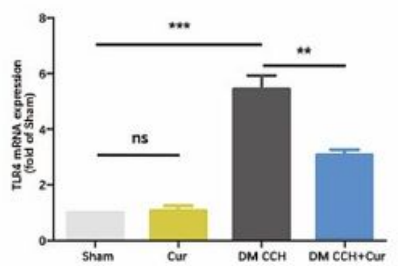

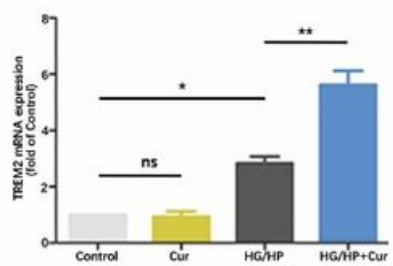

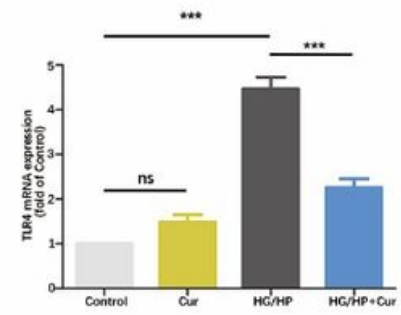

Figure 4

Curcumin regulates TREM2/TLR4/NF-KB signaling in DM/CCH rats and high-glucose/hypoxia-treated microglia. A, C Representative protein bands from Western blotting. B, D Quantitative analysis of Westernblot protein bands. E, F mRNA levels of TREM2 and TLR4. ${ }^{*} P<0.05 ; * \star P<0.01 ; * \star \star P<0.001$ Cur, Curcumin 
A

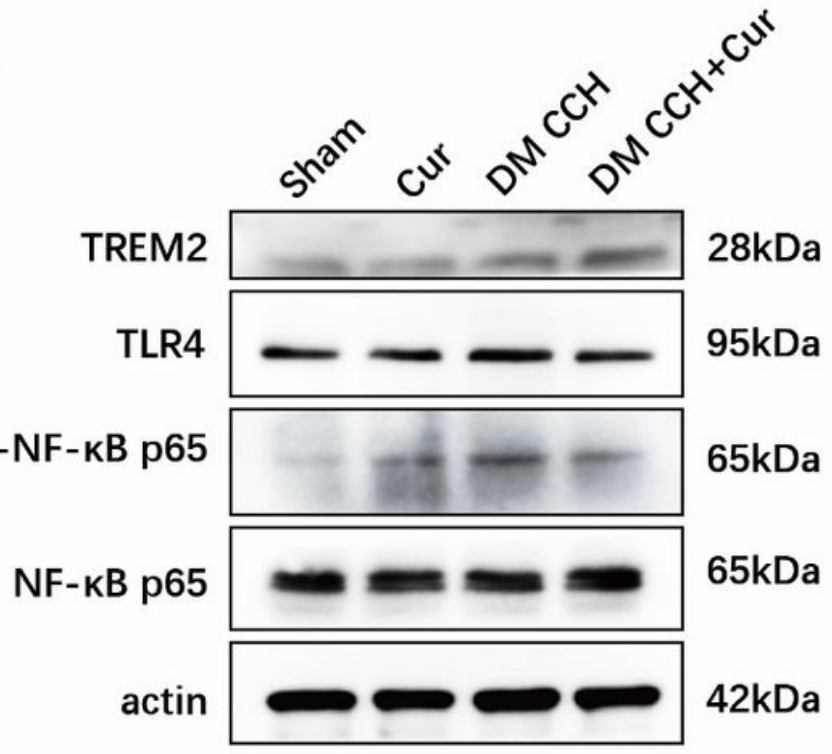

B
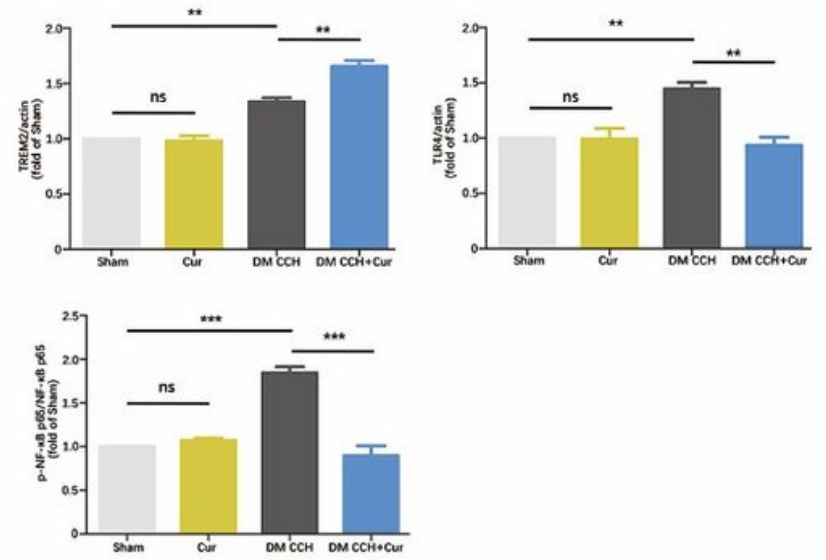

$E$

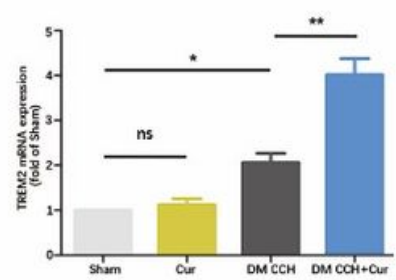

C

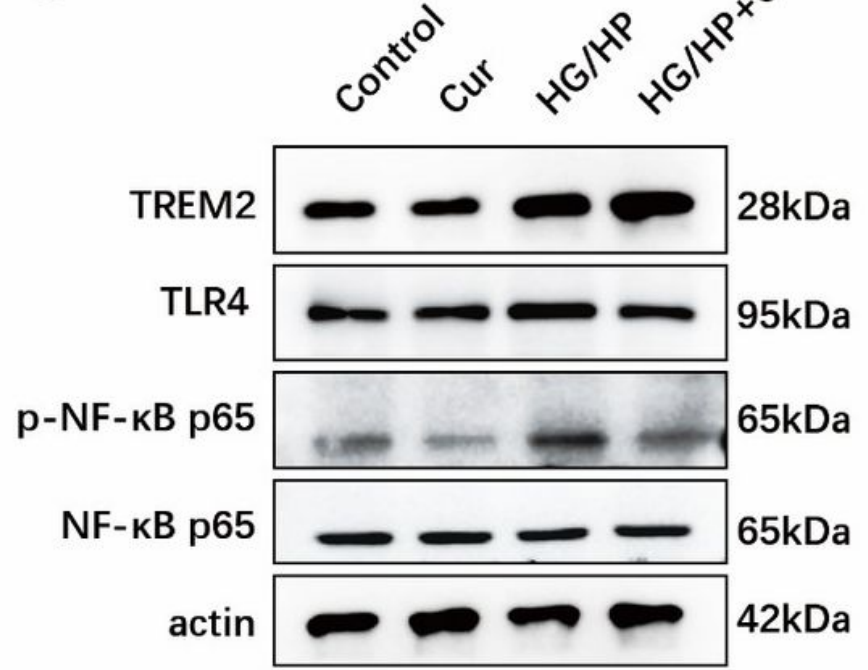

D
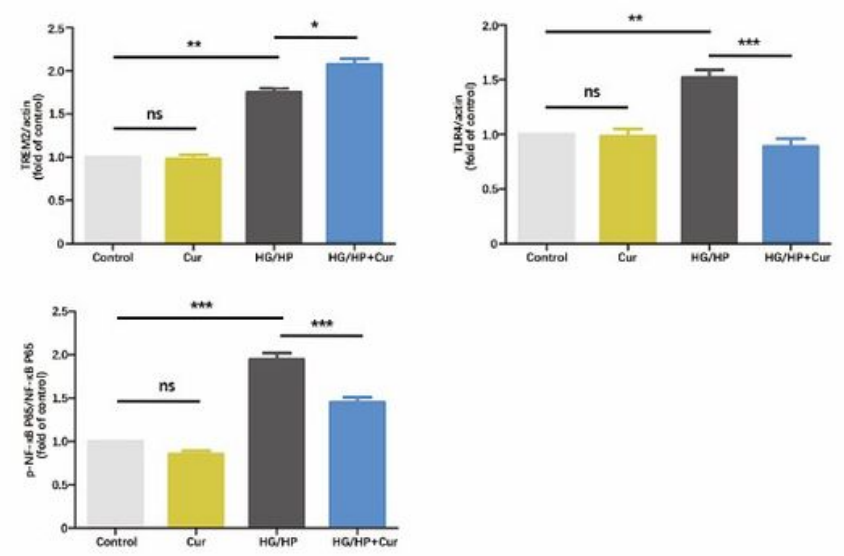

F

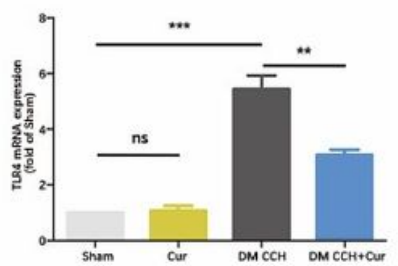

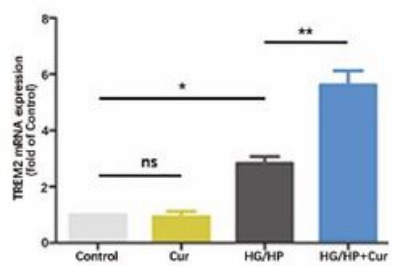

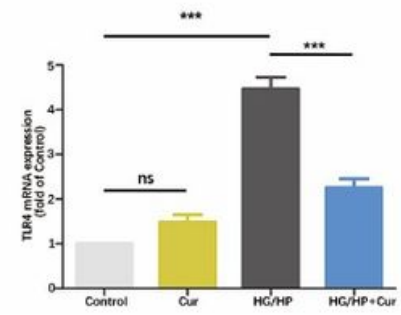

Figure 4

Curcumin regulates TREM2/TLR4/NF-KB signaling in DM/CCH rats and high-glucose/hypoxia-treated microglia. A, C Representative protein bands from Western blotting. B, D Quantitative analysis of Westernblot protein bands. E, F mRNA levels of TREM2 and TLR4. ${ }^{*} P<0.05 ; * \star P<0.01 ; * \star \star P<0.001$ Cur, Curcumin 
A

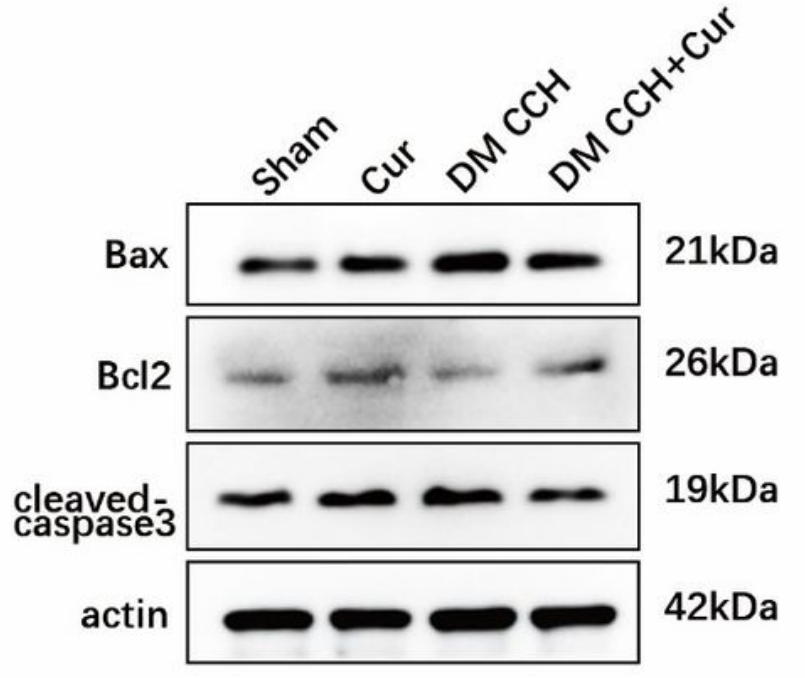

C

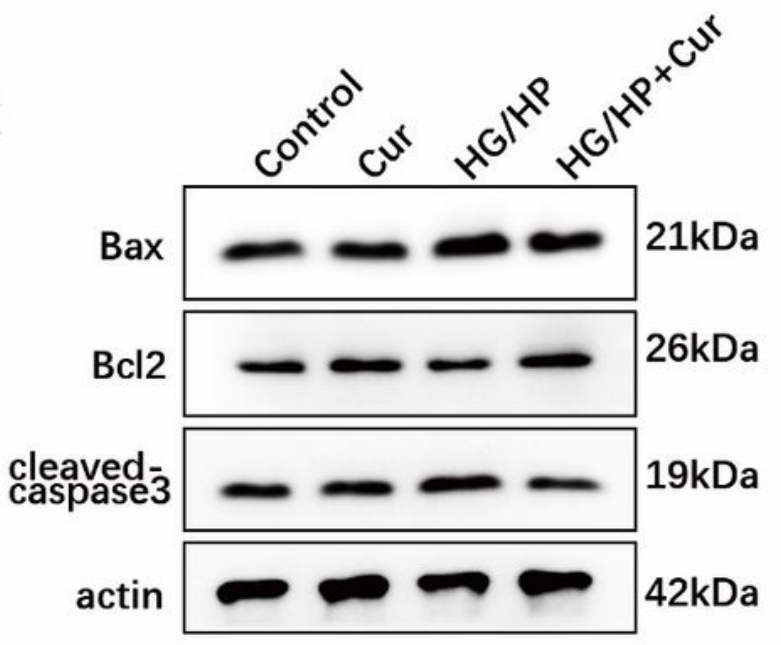

B

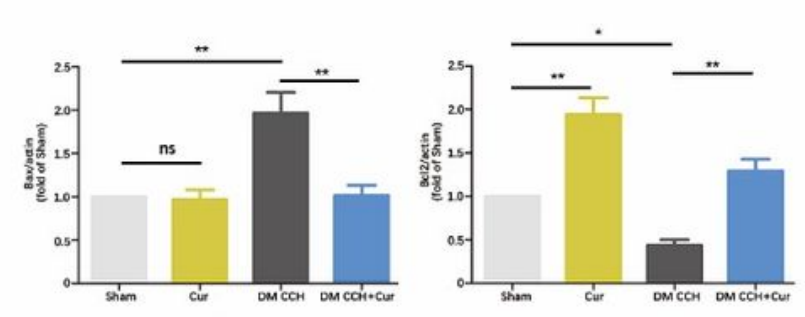

D
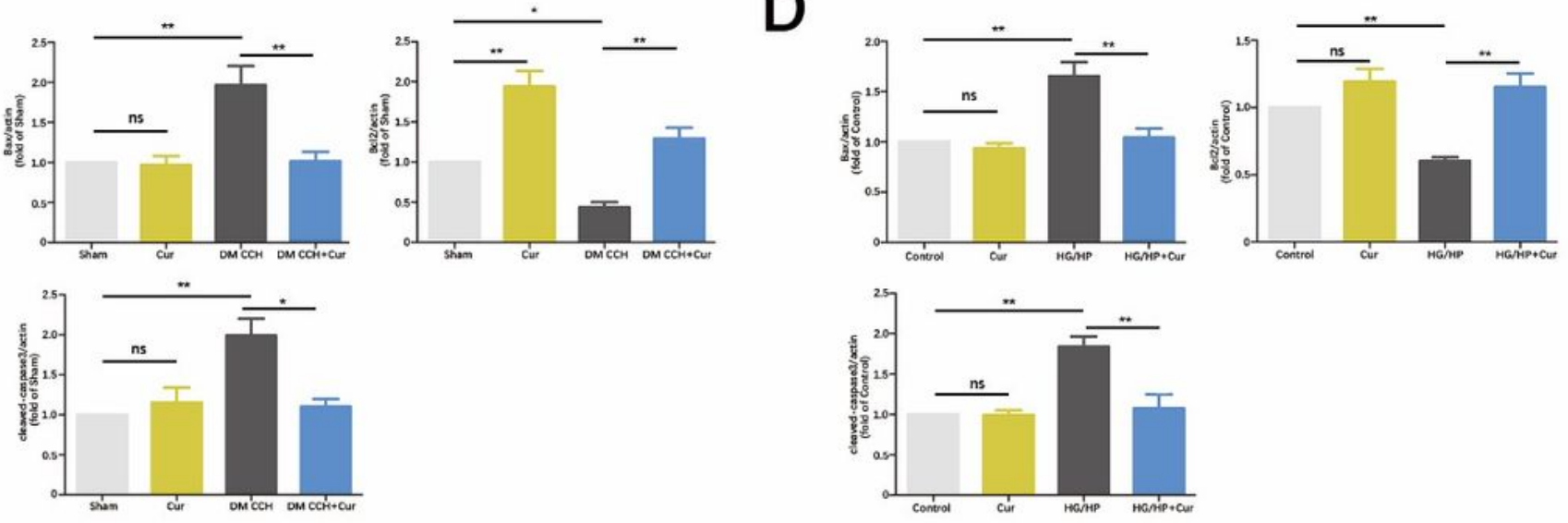

E
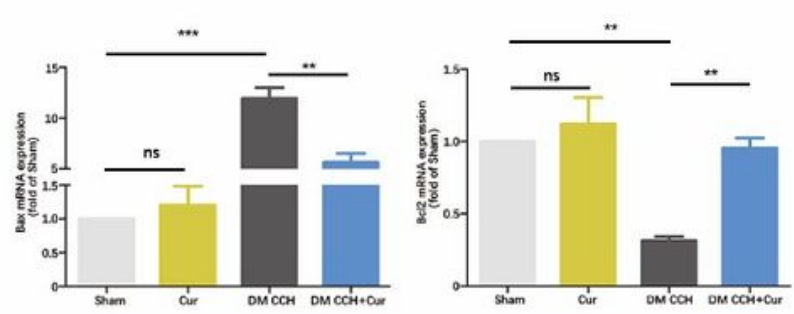

$\mathbf{F}$
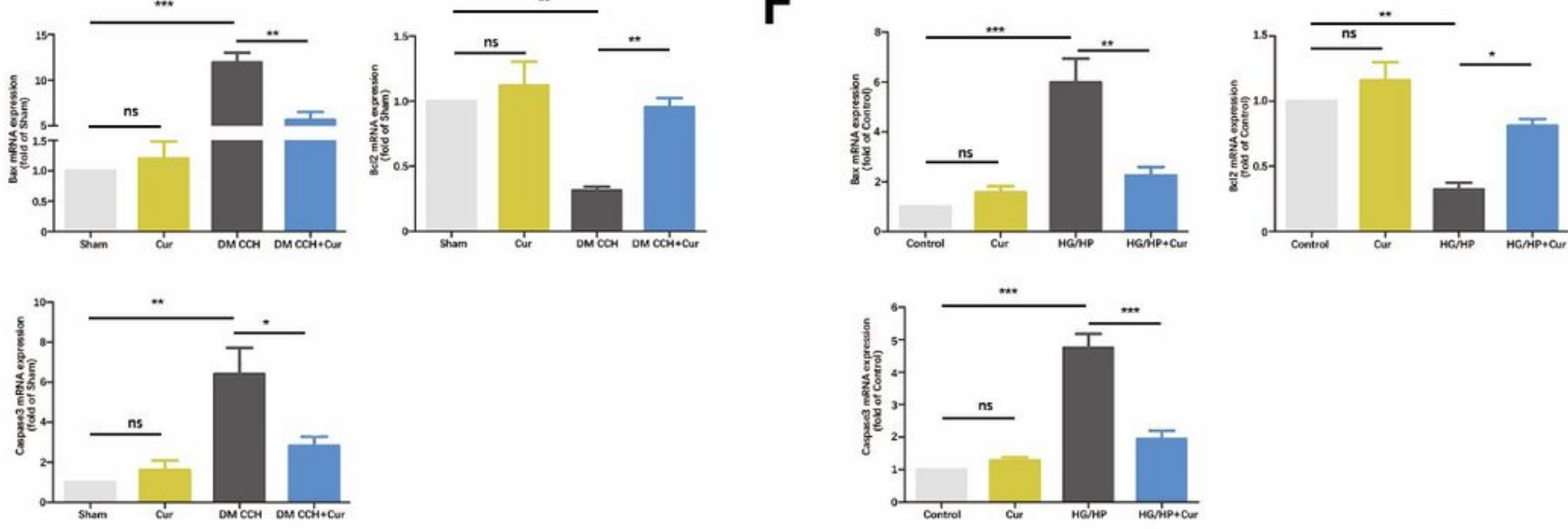

Figure 5

Curcumin suppresses apoptosis in $\mathrm{DM} / \mathrm{CCH}$ rats and high-glucose/hypoxia-treated microglia. A, B Representative protein bands from Western blotting. B, D Quantitative analysis of Western-blot protein bands. $\mathrm{E}, \mathrm{F}$ mRNA levels of $\mathrm{Bax}, \mathrm{Bcl} 2$, and Caspase3. ${ }^{\star} \mathrm{P}<0.05 ;{ }^{*} \mathrm{P}<0.01$; ${ }^{* \star \star} \mathrm{P}<0.001$ Cur, Curcumin 
A

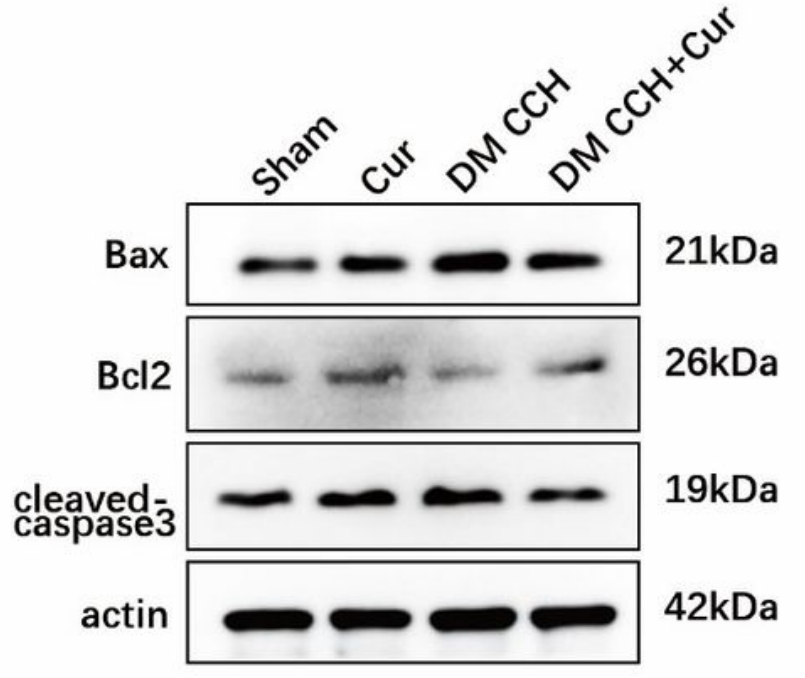

C

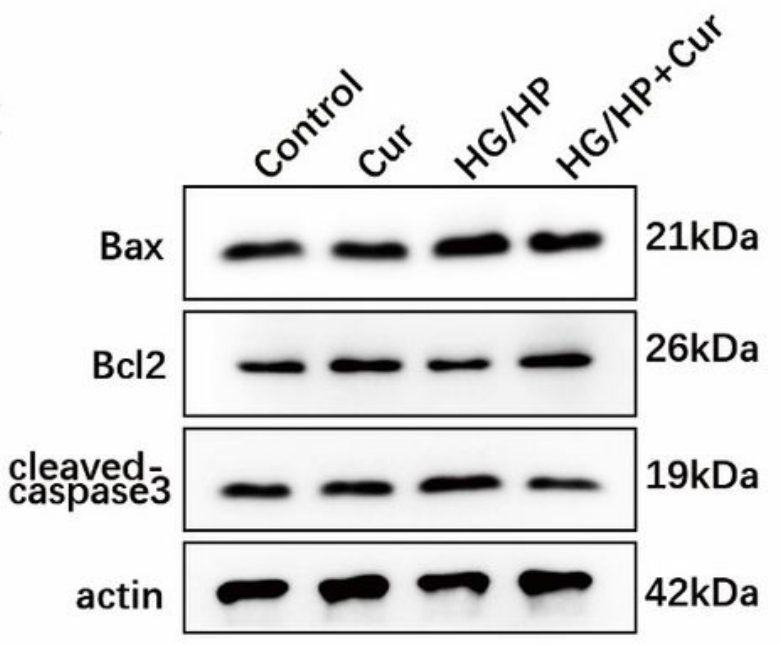

B

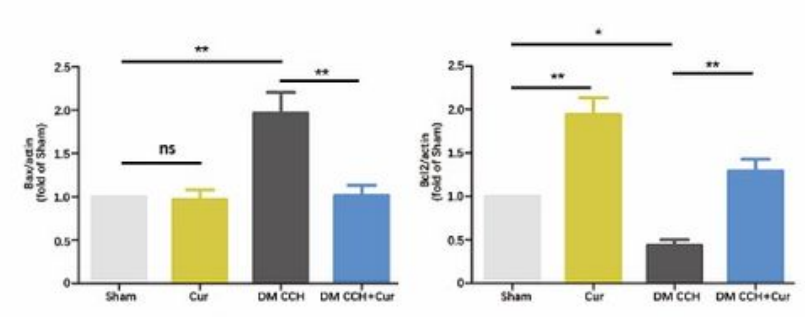

D
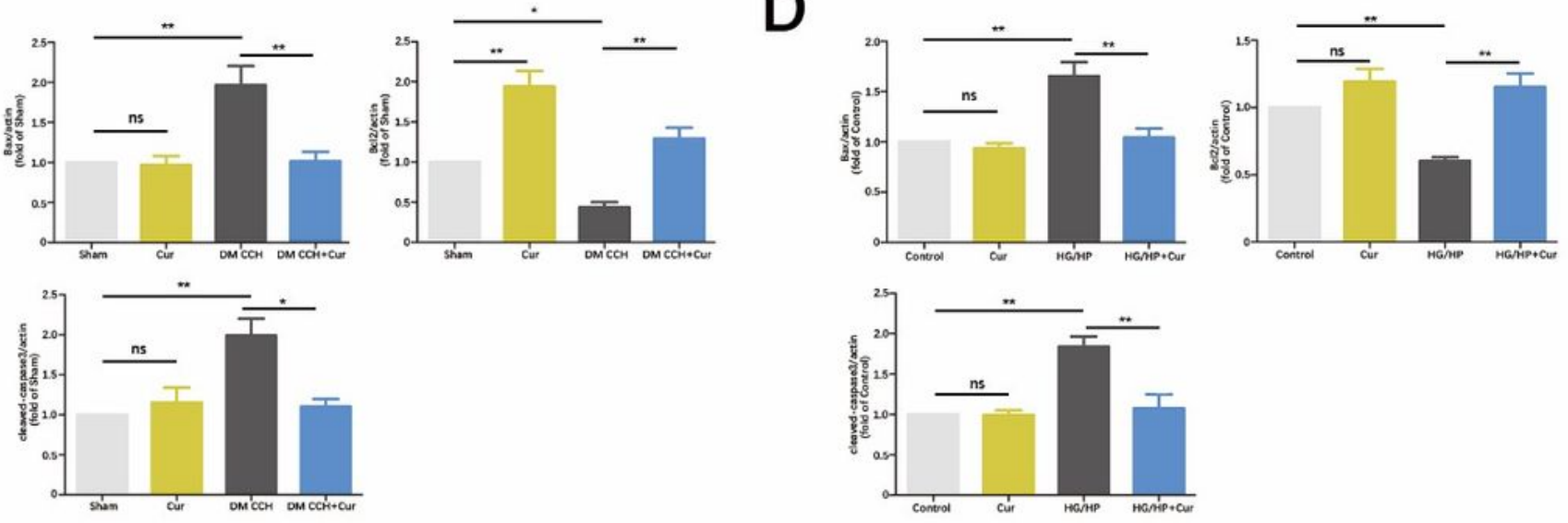

E
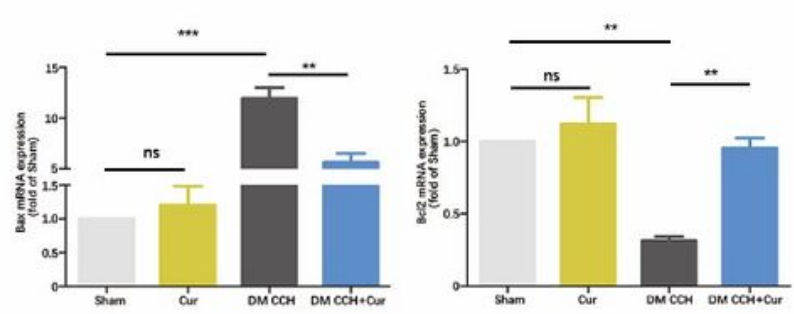

$\mathbf{F}$
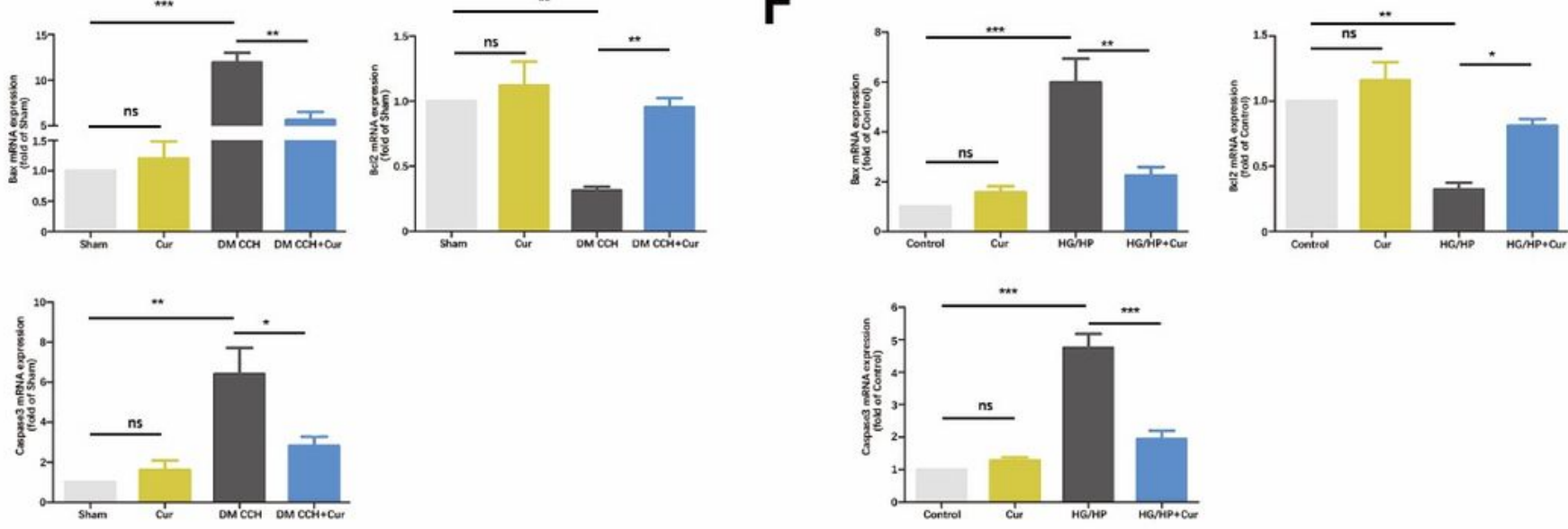

Figure 5

Curcumin suppresses apoptosis in $\mathrm{DM} / \mathrm{CCH}$ rats and high-glucose/hypoxia-treated microglia. A, B Representative protein bands from Western blotting. B, D Quantitative analysis of Western-blot protein bands. $\mathrm{E}, \mathrm{F}$ mRNA levels of $\mathrm{Bax}, \mathrm{Bcl} 2$, and Caspase3. ${ }^{\star} \mathrm{P}<0.05 ;{ }^{*} \mathrm{P}<0.01$; ${ }^{* \star \star} \mathrm{P}<0.001$ Cur, Curcumin 


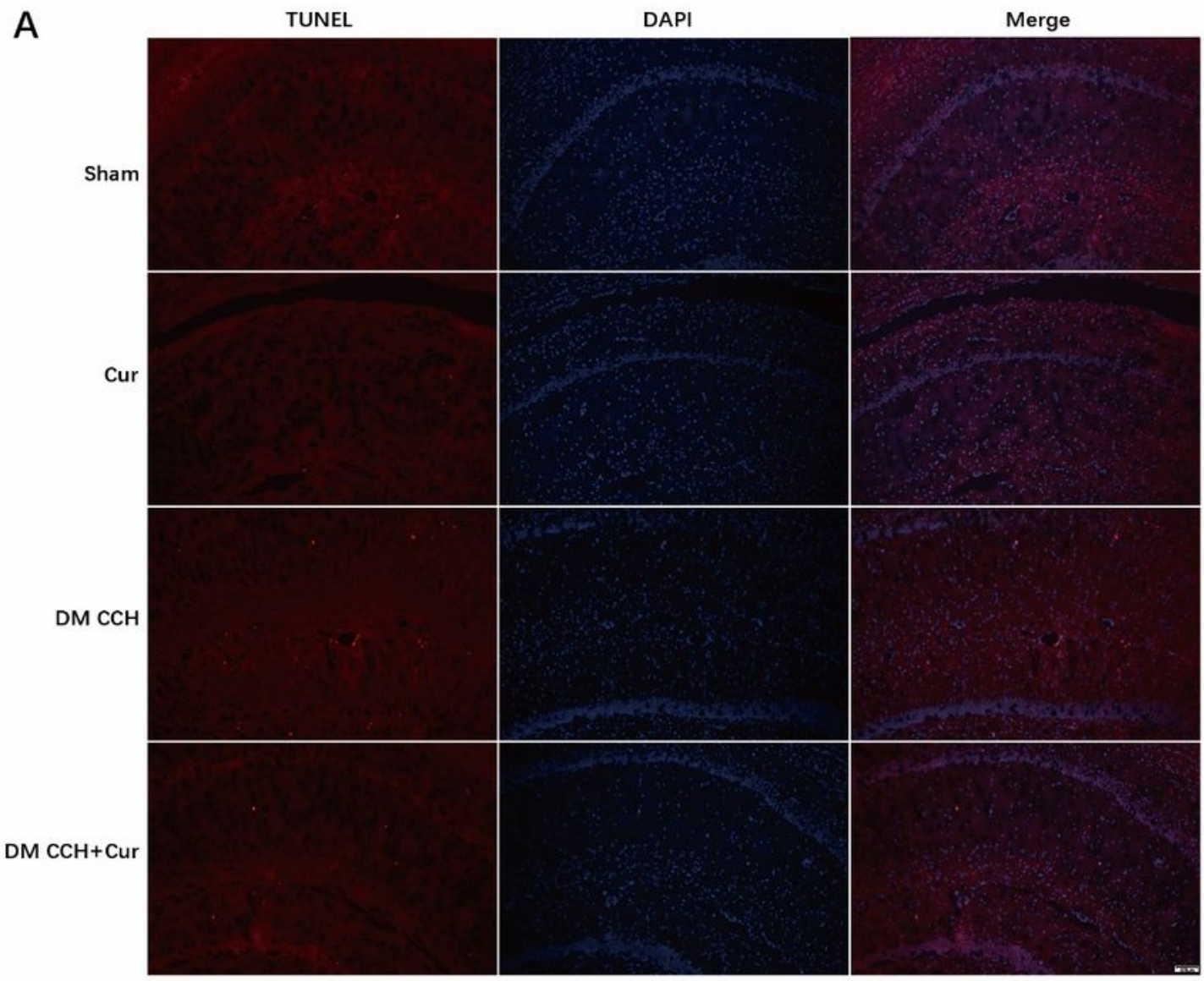

B
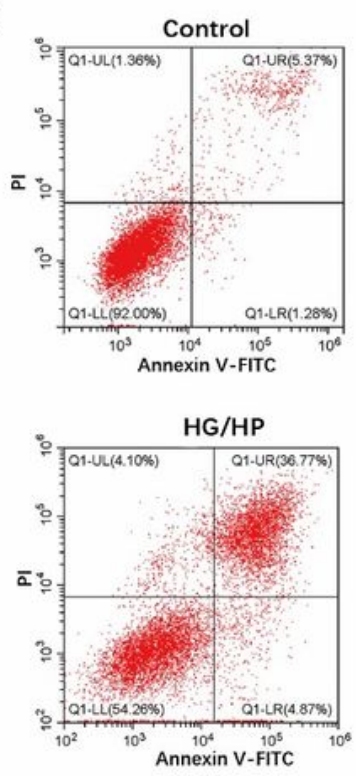
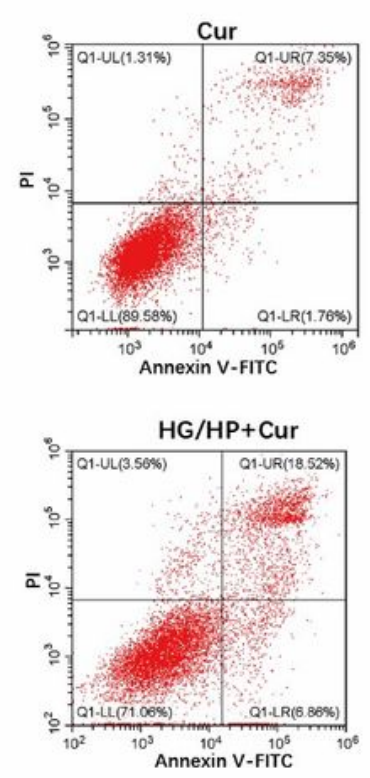

C

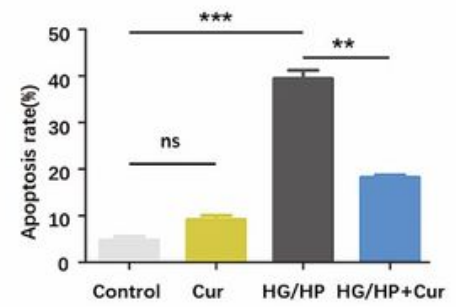

D

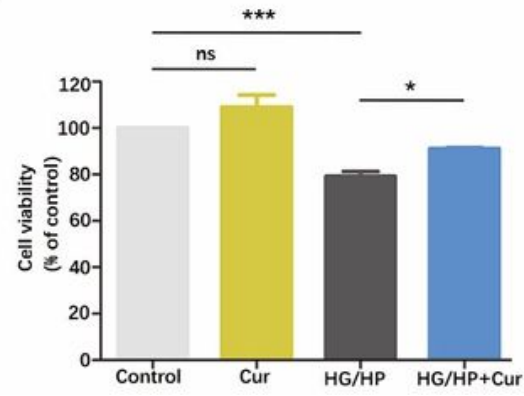

\section{Figure 6}

Curcumin suppresses apoptosis in $\mathrm{DM} / \mathrm{CCH}$ rats and high-glucose/hypoxia-treated microglia. A Representative photomicrographs showing apoptotic cells in the CA1 region of the hippocampus, as determined by TUNEL assays. B Numbers of apoptotic cells were measured by flow cytometry. C Quantitative analysis of apoptosis. D Cell viability was determined using CCK8 assays. ${ }^{*} \mathrm{P}<0.05$; $* \star \mathrm{P}<$ $0.01 ; * \star * P<0.001$ Cur, Curcumin 


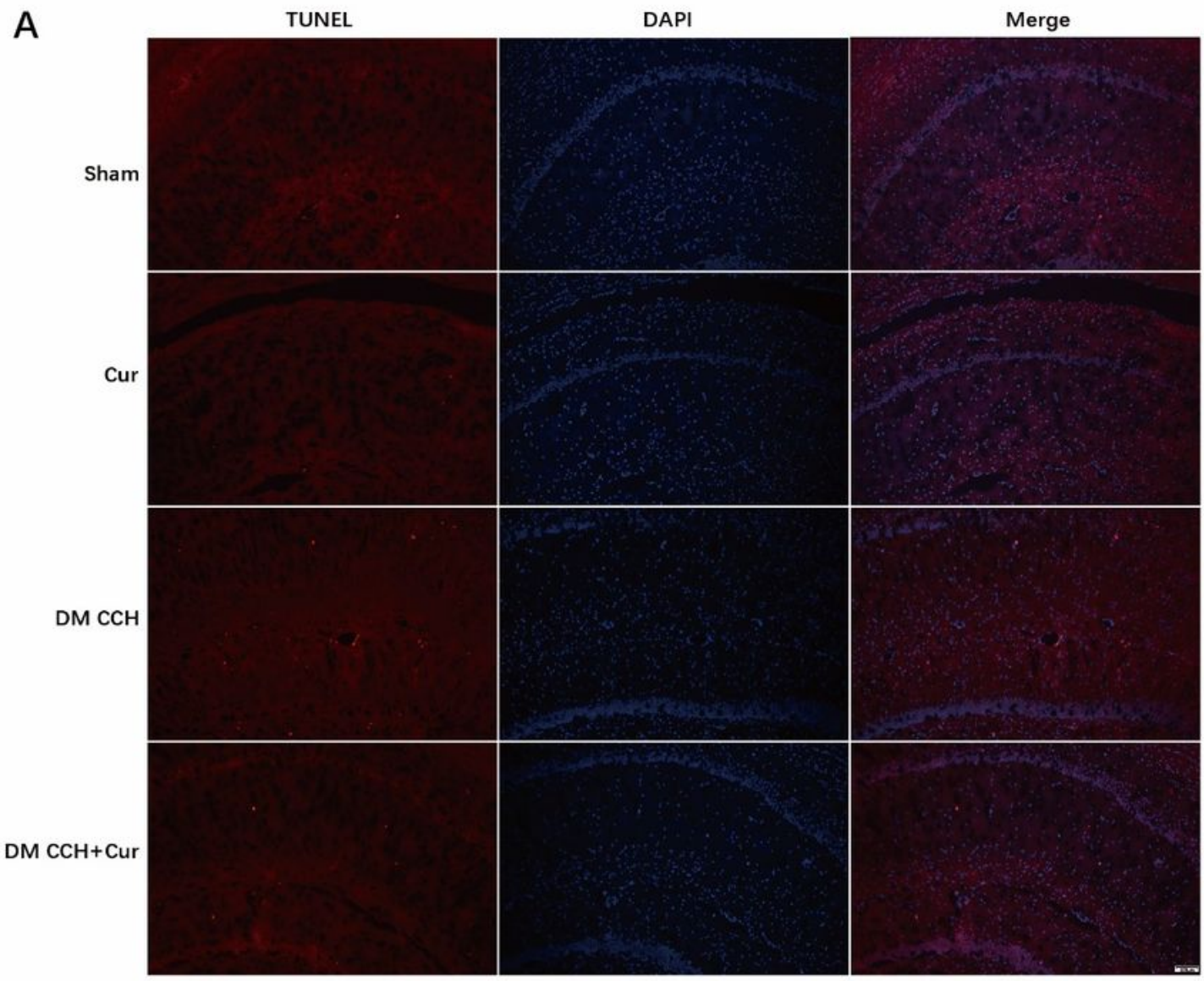

B
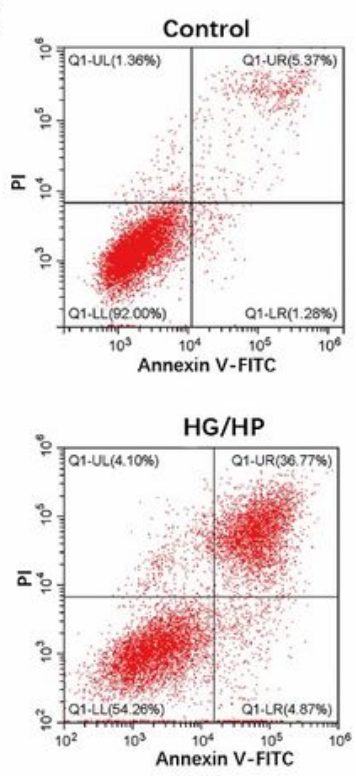
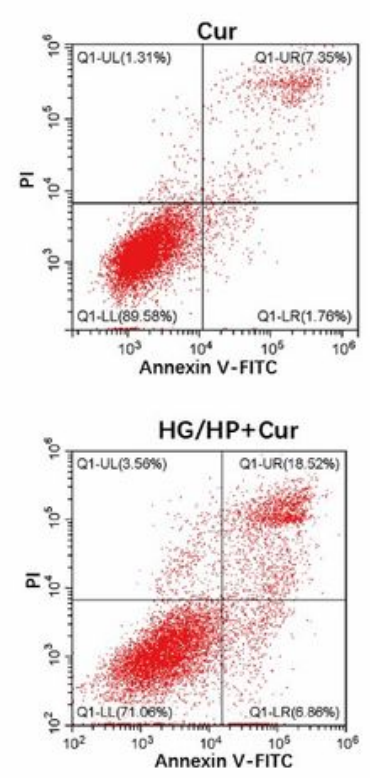

C

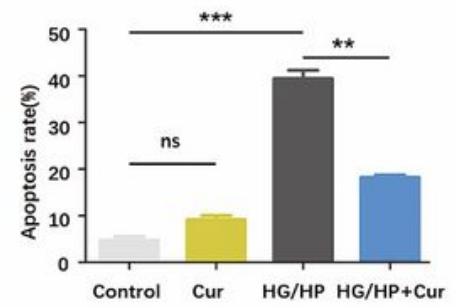

D

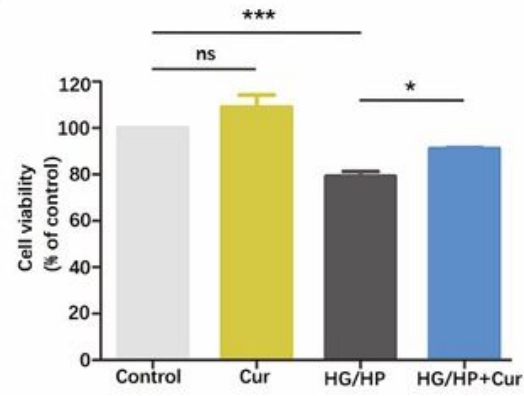

\section{Figure 6}

Curcumin suppresses apoptosis in $\mathrm{DM} / \mathrm{CCH}$ rats and high-glucose/hypoxia-treated microglia. A Representative photomicrographs showing apoptotic cells in the CA1 region of the hippocampus, as determined by TUNEL assays. B Numbers of apoptotic cells were measured by flow cytometry. C Quantitative analysis of apoptosis. D Cell viability was determined using CCK8 assays. ${ }^{*} \mathrm{P}<0.05$; $* \star \mathrm{P}<$ $0.01 ; * \star * P<0.001$ Cur, Curcumin 

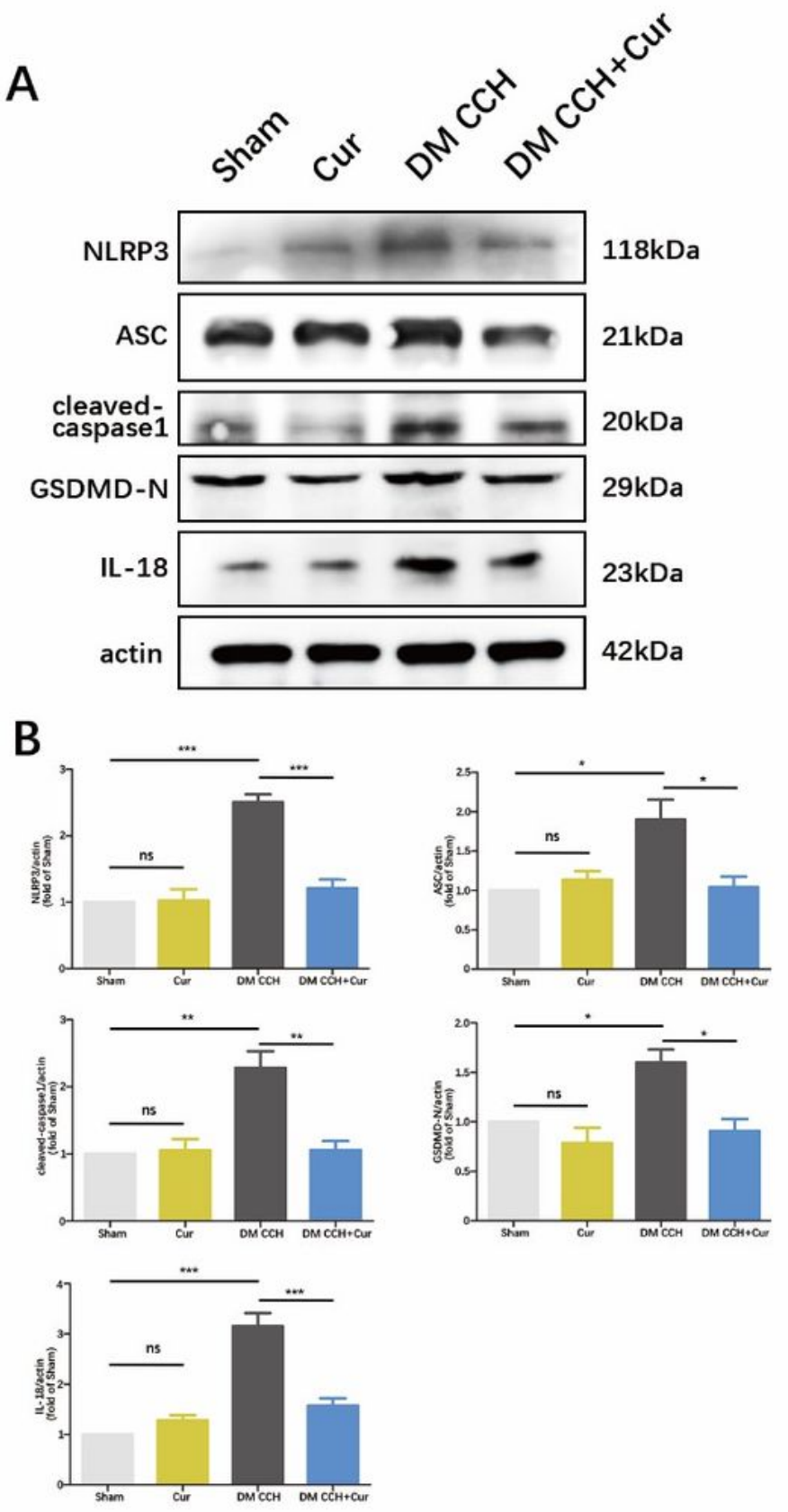

E
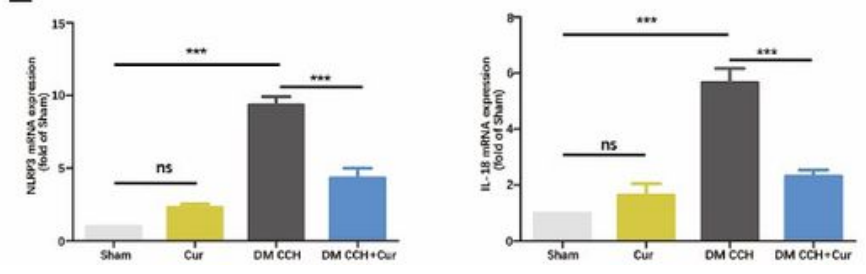

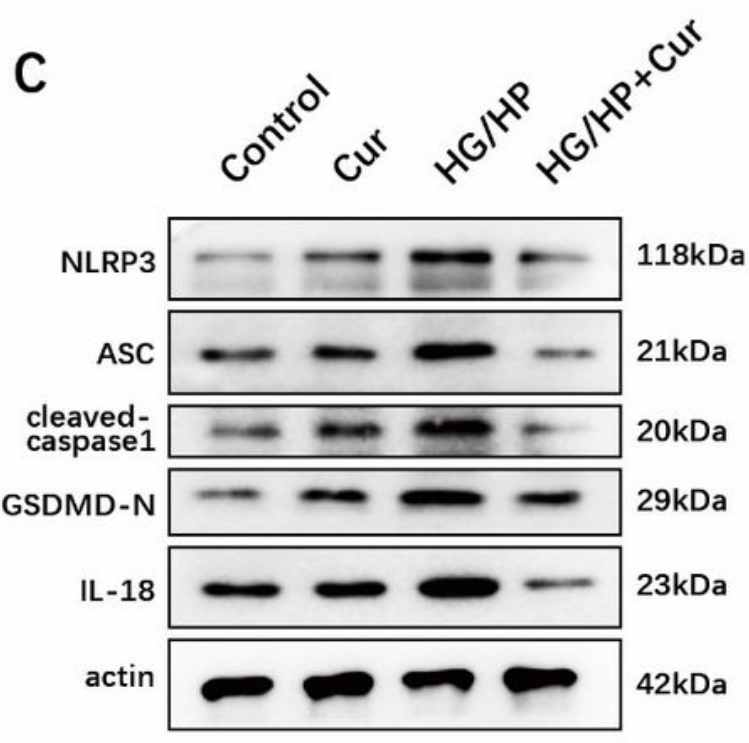

D
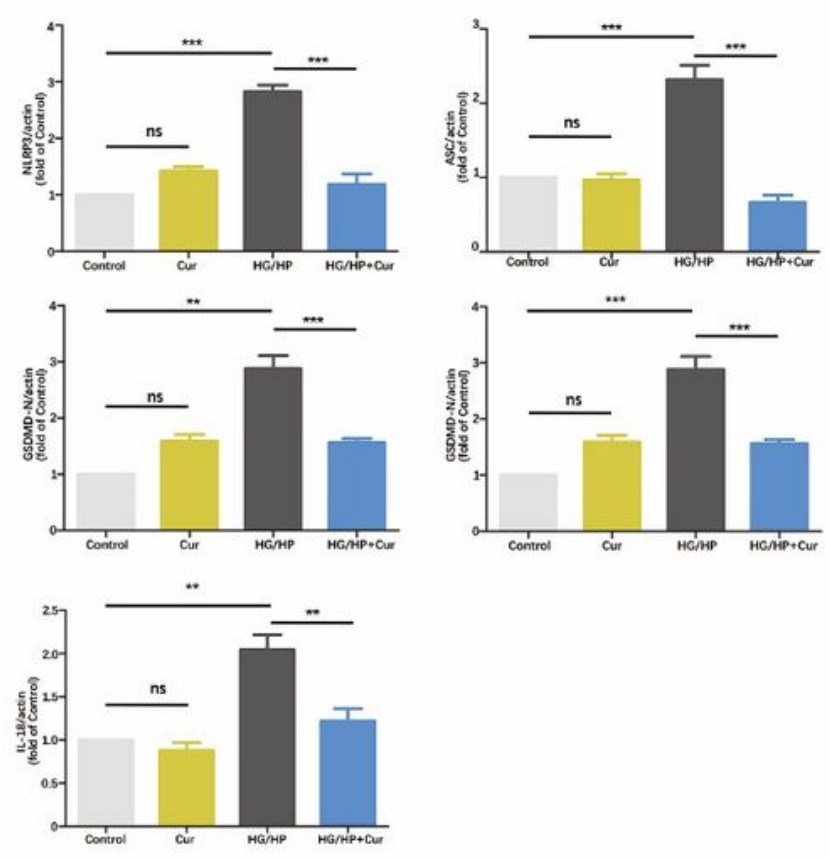

$\mathrm{F}$
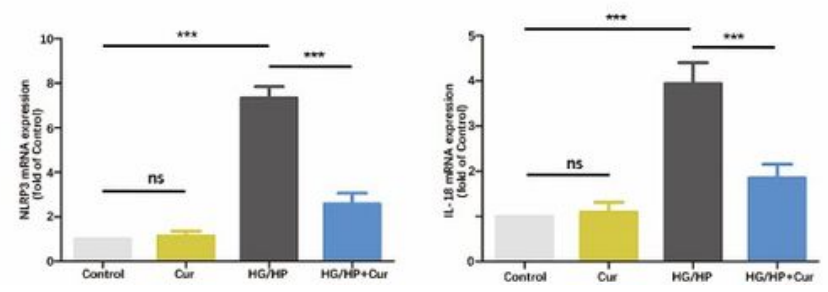

Figure 7

Curcumin mitigates NLRP3-dependent pyroptosis in DM/CCH rats and high-glucose/hypoxia-treated microglia. A, C Representative protein bands from Western blotting. B, D Quantitative analysis of Westernblot protein bands. E, F mRNA levels of NRLP3 and IL-18. ${ }^{\star} P<0.05 ;{ }^{* \star} \mathrm{P}<0.01$; ${ }^{* \star *} \mathrm{P}<0.001$ Cur, Curcumin 

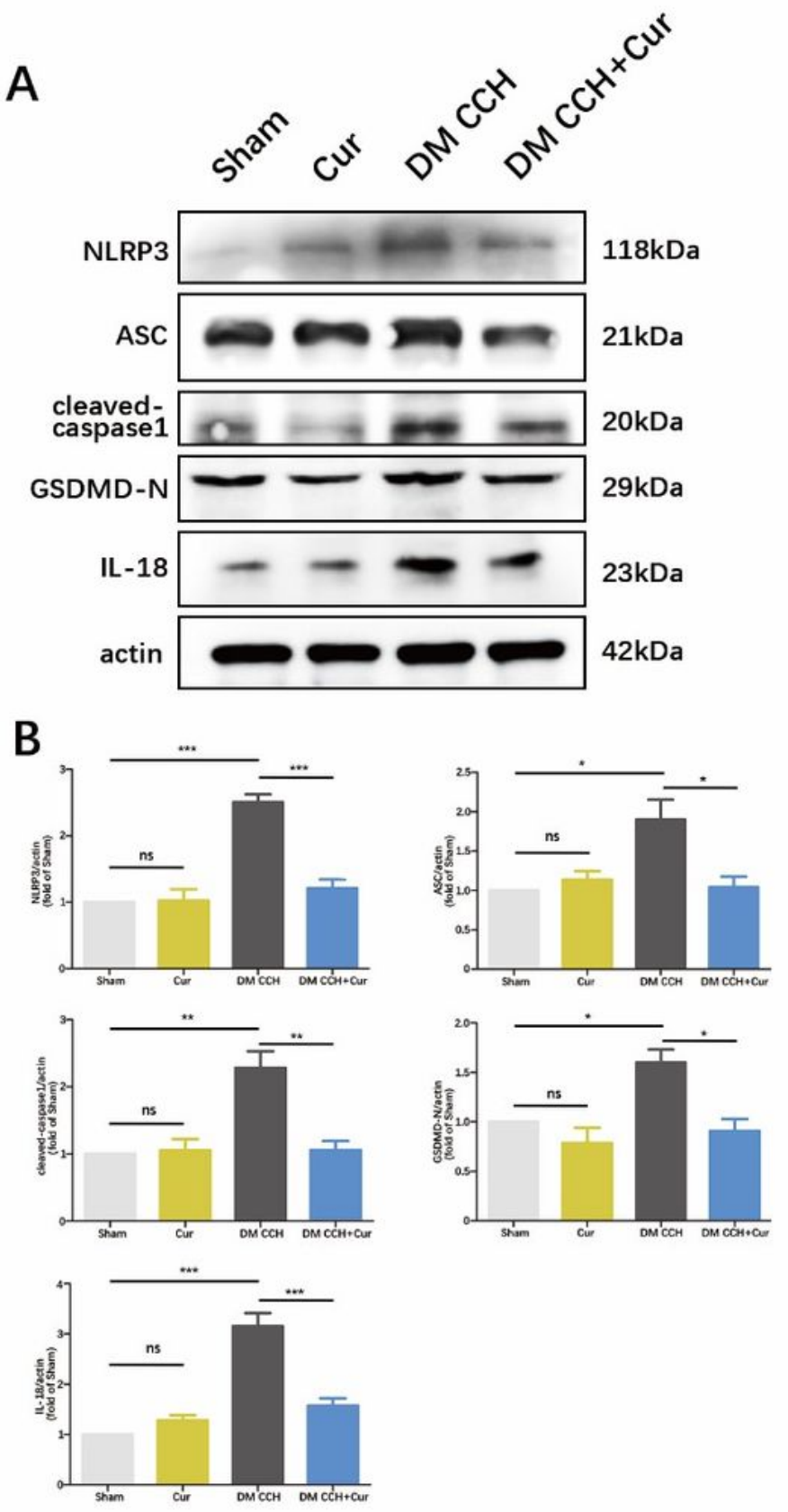

E
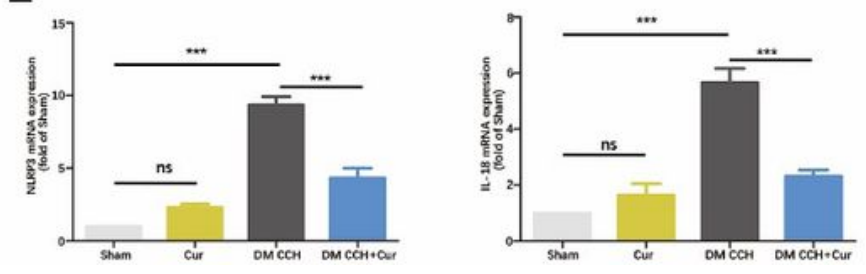

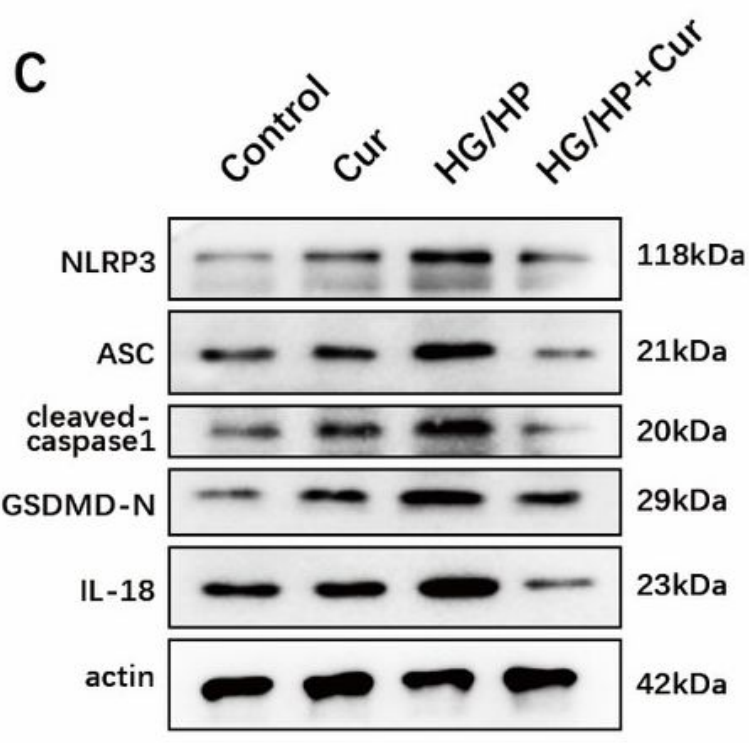

D
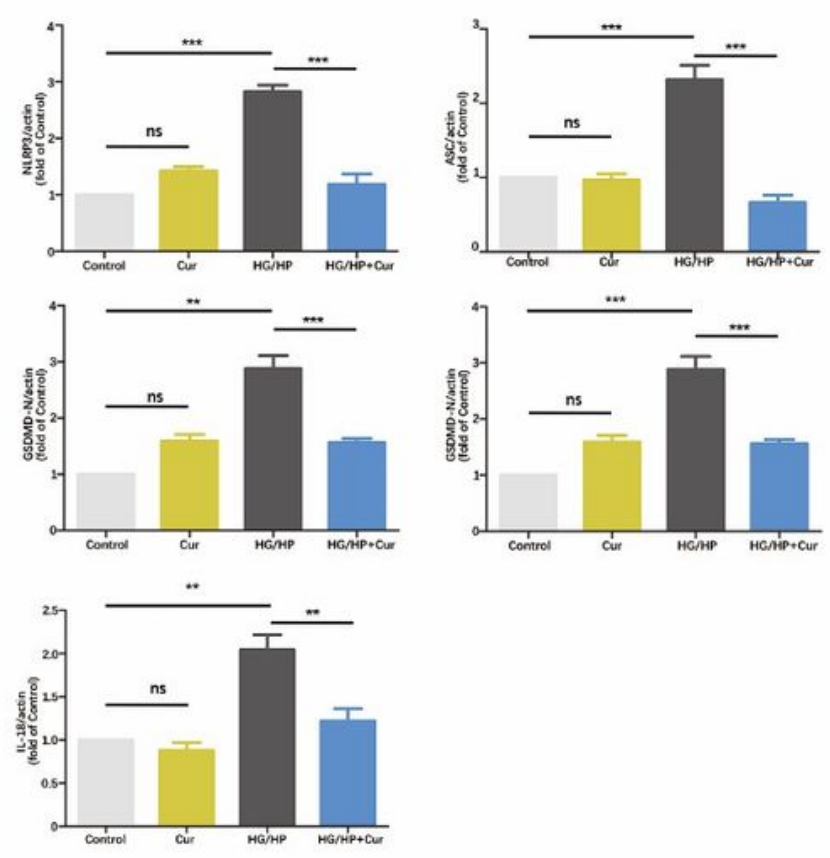

$\mathrm{F}$
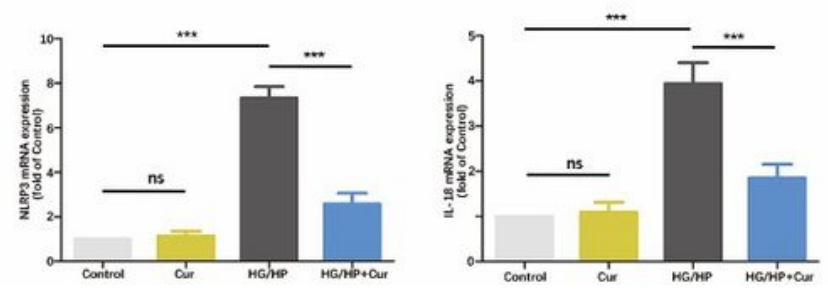

Figure 7

Curcumin mitigates NLRP3-dependent pyroptosis in DM/CCH rats and high-glucose/hypoxia-treated microglia. A, C Representative protein bands from Western blotting. B, D Quantitative analysis of Westernblot protein bands. E, F mRNA levels of NRLP3 and IL-18. ${ }^{\star} P<0.05 ;{ }^{* \star} \mathrm{P}<0.01$; ${ }^{* \star *} \mathrm{P}<0.001$ Cur, Curcumin 